\title{
Effect of Disordered Gut Microbiota on Serum Metabolome Alterations in Colorectal Tumor- Bearing Mice Under The Intervention of Fufangchangtai
}

\section{Mengmeng Cai}

Nanjing University of Chinese Medicine https://orcid.org/0000-0002-1403-8574

Zhibing Lin

Shanghai Jiao Tong University School of Agriculture and Biology

Ya Xiao

Nanjing University of Chinese Medicine

Jinmiao Lu

South China Agricultural University

Xiaoyu Wang

Shanghai Jiao Tong University School of Agriculture and Biology

Shilan Zhu

South China Agricultural University

Xiaoyu Chen

South China Agricultural University

Jialin $\mathrm{Gu}$

Nanjing University of Chinese Medicine

Yuzhu Ma

Nanjing University of Chinese Medicine

Zhaoguo Chen

Shanghai Veterinary Research Institute Chinese Academy of Agricultural Sciences

Jiege Huo ( $\nabla$ huojiege@jsatcm.com )

Nanjing University of Chinese Medicine https://orcid.org/0000-0002-0463-134X

Research

Keywords: Colorectal cancer, Fufangchangtai, Gut microbiota, Serum metabolome

Posted Date: April 26th, 2021

DOI: https://doi.org/10.21203/rs.3.rs-426536/v1 
License: (c) (i) This work is licensed under a Creative Commons Attribution 4.0 International License. Read Full License 
Effect of disordered gut microbiota on serum metabolome alterations in colorectal tumor-bearing mice under the intervention of Fufangchangtai

Mengmeng Cai, ${ }^{1,2,6}$ Zhibing Lin, ${ }^{3}$ Ya Xiao, ${ }^{1,2,4}$ Jinmiao Lu, ${ }^{5,6}$ Xiaoyu Wang, ${ }^{3}$ Shilan Zhu, ${ }^{5,6}$ Xiaoyu Chen, ${ }^{5,6}$ Jialin Gu, ${ }^{1,2,4}$ Yuzhu Ma, ${ }^{1,2,4}$ Zhaoguo Chen, ${ }^{6, *}$ Jiege Huo, ${ }^{2,4, *}$

${ }^{1}$ Nanjing University of Chinese Medicine, Nanjing, Jiangsu 210046, China

${ }^{2}$ Department of Oncology, Affiliated Hospital of Integrated Traditional Chinese and Western Medicine, Nanjing University of Chinese Medicine, Nanjing, Jiangsu 210028, China

${ }^{3}$ Shanghai Jiao Tong University School of Agriculture and Biology, Shanghai 201100, China

${ }^{4}$ Department of Oncology, Jiangsu Province Academy of Traditional Chinese Medicine, Nanjing, Jiangsu 210028, China

${ }^{5}$ South China Agricultural University, Guangzhou, Guangdong 510642, China

${ }^{6}$ Shanghai Veterinary Research Institute, Chinese academy of Agricultural Sciences, Shanghai

201100, China

Correspondence should be addressed to Jiege Huo and Zhaoguo Chen

Jiege Huo

huojiege@jsatcm.com

Affiliated Hospital of Integrated Traditional Chinese and Western Medicine, Nanjing University of Chinese Medicine, No. 100, Hongshan Road, Qixia District, Nanjing, Jiangsu 210028, China.

\title{
Zhaoguo Chen
}

zhaoguochen@shvri.ac.cn

Shanghai Veterinary Research Institute, Chinese academy of Agricultural Sciences, No.518, Ziyue Road, Minhang District, Shanghai 201100, China.

\begin{abstract}
:
Background: The occurrence and development of colorectal cancer is related to the compositional and functional variation of gut microbiota. Compared with healthy people, gut microbiota of patients with colorectal cancer is in disorder. Most traditional Chinese Medicine is effective by oral administration and has both anti-tumor effect and enteric microecological regulative effect. However, whether the dysbiosis of gut microbiota under tumor burden affects the serum metabolome of human body that related to traditional Chinese medicine is unclear. In this study, Fufangchangtai(FFCT) was chosen to be the model prescription to explore the correlation between gut microbiota and the serum metabolism related to FFCT in anti colorectal cancer treatment.
\end{abstract}


Results: The gut microbiota between colorectal tumor-bearing mice and healthy mice were determined by $16 \mathrm{~S}$ rRNA gene sequencing, showing quite differences between the two groups and suggesting that Firmicutes, Deferribacteres, Bacteroidetes and Proteobacteria were marked differential intestinal bacteria. The alternations in serum metabolome in the FFCT-treating tumor-bearing mice and simple FFCT-treating mice were detected using Liquid chromatogragh-mass spectrometer (LC/MS), showing significant differences between the two groups as well. Metabolites of FFCT like Citric acid, $( \pm)$ 12-HEPE, Cycloartanyl ferulate were much more in simple FFCT-treating mice, indicating that the present of tumor could affect the absorption and metabolism of FFCT. Additionally, these differential metabolites of FFCT involved in multiple pathways including the Alanine, aspartate and glutamate metabolism, Central carbon metabolism in cancer, Biosynthesis of amino acids. Different doses of FFCT were given to the tumor-bearing mice through oral administration, and the results of gut microbiota 16S rRNA gene sequencing showing that FFCT-treating groups has higher abundance of Firmicutes, Turicibacter and Roseburia than tumor-bearing group, moreover, the abundance of these bacteria was positively correlated to the drug concentration. Firmicutes and Bacteroidetes in FFCT-treating groups showed a similar trend with Healthy group, indicating the modulation of FFCT on gut microbiota of colorectal tumor-bearing mice.

Conclusions: Collectively, we concluded that the dysbiosis of gut microbiota in tumor-bearing mice could affect the serum metabolome of human body that related to FFCT, and FFCT could correct the gut microbiota of colorectal tumor-bearing mice. It was pointed out that the GM should be concerned during the therapy of FFCT. The more healthier intestinal microenvironment was conductive to the better clinical curative effect.

Keywords: Colorectal cancer, Fufangchangtai, Gut microbiota, Serum metabolome

\section{Introduction}

Colorectal cancer (CRC) is a malignant tumor that occurs to the colon or rectum. Although the death rate of $\mathrm{CRC}$ is decreasing continuously due to the population screening and endoscopic surveillance, it's incidence rate and mortality rate is still the third worldwide[1]. Gut microbiota (GM), which is called 'the new virtual metabolic organ'[2], plays a vital role in stimulating the digestion[3], producing nutrients [4], and promoting mucosal barrier immunity[5]. Once GM is out of balance, systematic diseases including carcinoma will occur. Many evidences indicated that there was a correlation between the development of CRC and dysbiosis of GM. Bacterial genes obtained by pyrosequencing of stool samples of healthy people and patients with CRC were subjected to the Principal Component Analysis (PCA) test. According to the PCA analysis, there 
were trends towards differences in the dominant and subdominant families of bacteria in CRC individuals and normal individuals[6]. GM initiates CRC by building tumoral microenvironment and inducing epithelial DNA damage and tumorigenesis, according to the 'diver-passenger' theory[7]. Meanwhile, GM also affects the immunity and prognosis of CRC patients by producing immunostimulatory and/or immunosuppressive cytokines such as IL-17A and IL-9[8]. Studies showed that tumor characterized by IL-17 expressing $\mathrm{T}$ cells, FOXP3hi Tregs and immunosuppressive myeloid populations were associated with worse clinical outcome[9].

There are many treatments for CRC, including radical surgical, chemotherapy, radiotherapy, targeted therapy, immunotherapy, etc. Correspondingly, these treatments have their own limitations and bring about many adverse reactions. Therefore, Traditional Chinese medicine (TCM) plays an important role in the prevention of tumor recurrence and metastasis due to its effect on synergy and attenuation. Chinese decoction such as Xiaoyaosan successfully reduced the tumor volume and tumor weight in mice with CRC xenografts and prolonged the overall survival time through a 21-days intragastrical treatment[10]. Herb such as Matricaria chamomilla could act as a potent single component against DMH-induced CRC by modulating the Wnt pathway[11]. Fufangchangtai (FFCT) is a prescription that has an effect on treatment of CRC by enhancing the body's immune, improving the patient's quality of life and down-regulating the side effects, which had been proved by clinical observation [12].

Most TCM is taken orally and absorbed into the blood through the gastrointestinal tract, thus exerting its efficacy. Therefore, TCM can reverse or mitigate varied compositional dysbiosis of GM associated with many diseases besides improving the pathological symptoms[13]. Study showed that Berberine rescued $F$. nucleatum-induced colorectal tumorigenesis by modulating the tumor microenvironment and blocking the activation of tumorigenesis-related pathways[14]. About TCM formula, Yi-Yi-Fu-Zi-Bai-Jiang-San (YYFZBJS) blocked tumor initiation in treating CRC by mediating Tregs and regulating the natural GM of Apc (Min/+) mice including Bacteroides fragilis, Lachnospiraceae and so on[15]. GM harbors many types of enzymes, allowing plenty of catalytic reactions, which are involved in the biotransformation of TCM components. Though many investigations indicated that GM could interact complicatedly with TCM components, whether GM could influence the therapeutic effect of TCM on CRC still remains unknown.

Thus, there is the speculation that the dysbiosis of GM affects the serum metabolome of human body that related to TCM in treating CRC. To test the hypothesis, this study chose FFCT as the model prescription. Different doses of FFCT were given to CRC tumor-bearing mice. The 16S rRNA gene sequencing technology was applied to analysis the bacteria species in the fecal 
samples, and the Liquid Chromatography Coupled to Tandem Mass Spectrometry (LC/MS) technology was applied to analysis the metabolites in the blood. The above data were analyzed to explore the correlation between GM and the metabolism of TCM in CRC treatment.

\section{Materials and methods}

\subsection{Preparation of drugs}

FFCT was formed by 6 kinds of TCM granules, including Ginseng, Membranous milkvetch root, Radix Actinidiae chinensis, August letters, Raw coix seed, Radix sophorae flavescentis, which were purchased from Jiangsu Hospital of integrated traditional Chinese and Western Medicine. The quality of formula was monitored by HPLC analysis in our previous study[16]. The formula granules were milled to powders and dissolved in warm distilled water to giving to mice.

\subsection{Cell culture and injection}

CT26-LUC cells were provided by Shanghai Institute of Veterinary Medicine and cultured with RPMI-1640 containing 10\% fetal bovine serum, $1 \%$ penicillin/streptomycin, and $4 \mu \mathrm{g} / \mathrm{ml}$ puromycin dihydrochloride in an incubator. The condition of the incubator was stable, with the temperature of $37^{\circ} \mathrm{C}$ and a humidified atmosphere with the $5 \% \mathrm{CO} 2$.

\subsection{Experimental Animals}

Six-week-old female BALB/C mice (ethics number: SHVRI-SZ-20200420-01, SHVRI-SZ-20200720-01) were purchased from Shanghai Jiesijie Company (Shanghai, China) and housed under a specific pathogen-free (SPF) condition with $25 \pm 2^{\circ} \mathrm{C}$ temperature and a 12 -h light/dark cycle. The mice were freely accessed a standard rodent diet and drinking water.

\subsubsection{Modeling and groups}

After one week adaption of the new environment, these mice were randomly divided into six groups: the PBS group (normal mice administered with PBS, $n=4$ ), the CM group (normal mice administered with FFCT, $\mathrm{n}=5$ ), the $\mathrm{CT} 26$ group ( $\mathrm{CRC}$ tumor-bearing mice administered with PBS, $\mathrm{n}=5$ ), the CT26-L group (CRC tumor-bearing mice administered with $0.65 \mathrm{mg} / \mathrm{g} F F C T, n=5)$, the CT26-M group (CRC tumor-bearing mice administered with $1.3 \mathrm{mg} / \mathrm{g}$ FFCT, $\mathrm{n}=5$ ), the $\mathrm{CT} 26-\mathrm{H}$ group (CRC tumor-bearing mice administered with $2.6 \mathrm{mg} / \mathrm{g}$ FFCT, $\mathrm{n}=5$ ). The daily dosage for the CT26-M group was obtained based on the daily dosage for patients $(10 \mathrm{~g} / 70 \mathrm{~kg})$ in clinical, according to the human-mouse transfer formula (Mouse dose=Human dose $\times 9.1[17]$ ). Before administration, CT26-LUC cells $\left(1 \times 10^{6}\right)$ were resuspended in $100 \mu$ of PBS and the suspension was inosulated subcutaneously into the flanks of mice in CRC tumor-bearing groups, while pure $100 \mu 1$ PBS was inosulated subcutaneously into the flanks of mice in the PBS group and the CM group. $200 \mu \mathrm{l}$ PBS or FFCT suspension was given to mice via gastric gavage, and the 
administration lasted for 4 weeks.

\subsubsection{Samples collection}

Faecal collection: Stools were collected from the mice the day before sacrificing mice. The mice were separated in cage individually and the clean toilet paper was laid under the cage to accept their faecal pellets and absorb the urine, avoiding contamination of samples. 2-4 faecal pellets per mice were collected in $1.5 \mathrm{ml} \mathrm{Ep}$ tube and stored at $-80^{\circ} \mathrm{C}$ until analyzed.

Blood collection: Drawing the blood from the eyeball of mice after the last oral administration $24 \mathrm{~h}$ and keeping the blood static cool down at $4^{\circ} \mathrm{C}$ for $1 \mathrm{~h}$. Then the serum was separated through centrifugation in $3000 \mathrm{rpm}$ for $5 \mathrm{~min}$ at $4^{\circ} \mathrm{C}$ and stored at $-80^{\circ} \mathrm{C}$ until analyzed.

\section{$2.4 \mathrm{GM}$ analysis}

Nucleic acid sequence of the bacterial 16S rRNA gene has been used for several decades to identify routine identification of bacterial pathogens[18].The 16S rRNA gene sequencing technology was applied to detect the V3-V4 region of bacterial species in twenty-four stool samples from five groups (PBS, CT26, CT26-L, CT26-M, CT26-H) and was entrusted to Personal Bio company.

The OMEGA Soil DNA Kit (D5625-01) (Omega Bio-Tek, Norcross, GA, USA) was used to extract the DNA from fecal samples. 16S rRNA amplicons covering variable regions V3 to V4 were generated using the forward primers 338F (5'-ACTCCTACGGGAGGCAGCA-3') and the reverse primer 806R (5'-GGACTACHVGGGTWTCTAAT-3'). PCR amplicons were purified with Vazyme VAHTSTM DNA Clean Beads (Vazyme, Nanjing, China) and quantified using the Quant-iT PicoGreen dsDNA Assay Kit (Invitrogen, Carlsbad, CA, USA). Then, amplicons were sequenced using the Illumina MiSeq platform with MiSeq Reagent Kit v3 at Shanghai Personal Biotechnology Co., Ltd (Shanghai, China). Sequence data analyses were mainly performed using QIIME2 and R packages (v3.2.0).

\subsection{Blood metabolomics analysis}

Metabolites are stable in serum and can be quantified, which presents an opportunity in monitoring disease status and exploring biomarkers to predict the efficacy of anticancer therapies[19]. The blood metabolomics of four groups (PBS, CM, CT26, CT26-H) were detected. The blood was drawn from the eyeball of mice and then stood at $4{ }^{\circ} \mathrm{C}$ for 1 hour. After $15 \mathrm{~min}$ centrifugation of $3000 \mathrm{rpm}$ at $4{ }^{\circ} \mathrm{C}$, the supernatant was collected and stored in dry ice for blood mass spectrometry. Liquid chromatogragh-mass spectrometer analysis of blood was entrusted to Luming Bio company.

$100 \mu \mathrm{l}$ of serum from each sample was eddied with $10 \mu \mathrm{l}$ internal standard $(0.3 \mathrm{mg} / \mathrm{ml}$ L-2-chlorophenylalanine or $0.01 \mathrm{mg} / \mathrm{ml}$ Lyso PC 17:0 dissolved in methanol ) for $10 \mathrm{~s}$. The 
mixtures were precipitated by $300 \mu \mathrm{l}$ mixtures of methanol and acetonitrile $(2 / 1, \mathrm{v} / \mathrm{v})$ and then were ultrasonicated in ice water bath for $10 \mathrm{~min}$, stored at $-20^{\circ} \mathrm{C}$ for $30 \mathrm{~min}$. After $10 \mathrm{~min}$ centrifugation (13000rpm, $4^{\circ} \mathrm{C}$ ), $300 \mu \mathrm{l}$ supernatant of each sample was collected and dried. The dried supernatant was resolved in $400 \mu \mathrm{l}$ of $20 \%$ methanol (diluted by water) and then placed at $-20^{\circ} \mathrm{C}$ for $2 \mathrm{~h}$. Times up, $150 \mu \mathrm{l}$ supernatant per sample was filtered through $0.22 \mu \mathrm{m}$ microfilter and transferred into LC sampling vial for LC/MS analysis. Additionally, the quality control (QC) sample was created by mixing an aliquot of equal volume of each sample.

A Dionex Ultimate 3000 RS UHPLC system (Thermo Fisher Scientific, USA) coupled with Q-Exactive quadrupole-Orbitrap mass spectrometer (Thermo Fisher Scientific, USA) was used to analyze the metabolic profiling in both ESI positive and ESI negative ion modes. $2 \mu$ prepared sample was injected into ACQUITY UPLC HSS T3 column $(1.8 \mu \mathrm{m}, 2.1 \times 100 \mathrm{~mm})$. All samples were eluted using a linear gradient from $100 \%$ mobile phase A $(0.1 \%$ formic acid in water) to $100 \%$ mobile phase B $(0.1 \%$ formic acid in acetonitrile) under the condition that the flow rate was $350 \mu \mathrm{l} / \mathrm{min}$ and the column temperature was $45^{\circ} \mathrm{C}$. Linear gradient: 0 min, $5 \% \mathrm{~B} ; 2 \mathrm{~min}, 5 \% \mathrm{~B}$; $4 \min , 25 \% \mathrm{~B}$; $8 \mathrm{~min}, 50 \% \mathrm{~B}$; $10 \mathrm{~min}, 80 \% \mathrm{~B}$; $14 \mathrm{~min}, 100 \% \mathrm{~B}$; $15 \mathrm{~min}, 100 \% \mathrm{~B} ; 15.1 \mathrm{~min}, 5 \% \mathrm{~B}$ and $16 \mathrm{~min}, 5 \% \mathrm{~B}$. The electrospray ionization (ESI) source operating in positive and negative mode (Water, USA) was used for mass spectrometry analysis. Parameters of mass spectrometry were as follows: Capillary temperature was set at $320^{\circ} \mathrm{C}$ while the aux gas heater temperature was set at $350^{\circ} \mathrm{C}$. Sheath gas flow rate was $35 \mathrm{Arb}$ and aux gas flow rate was $8 \mathrm{Arb}$. The san range was from 100 to $1000 \mathrm{~m} / \mathrm{z}$.

The raw data was processed by the Progqenesis QI v2.3 software (Nonlinear Dynamics, Newcastle, UK) for baseline filtering, peak recognition, peak alignment and retention time correction. The Human Metabolome Database (HMDB), Lipidmaps (v2.3) and METLIN database and self-built database were used to identify the compounds.

\section{Results}

3.1 There were differences in GM between CRC tumor-bearing mice and healthy mice

There is increasing evidence that GM in CRC patients is different from that in healthy people. The Faecal microbiota of CRC patients has lower temporal stability and higher diversity[20], and an individualised oncogenic microbiome and specific bacterial species have been identified through metagenomic and meta transcriptomic studies[21]. Streptococcus gallolyticus, Enterococcus faecalis and B. fragilis, et al, are considered as specific pathobionts with CRC[22]. To prove the difference in GM, the faecal of CRC tumor-bearing mice were used to detect the microbiota comparing with that of healthy mice.

The GM of five groups (PBS, CT26, CT26-L, CT26-M, CT26-H) was analyzed. The 
rarefaction curves of five groups (Figure 1) were close to the saturation platform at the sequencing depth of 10000, indicating that the data was sufficient enough to reflect the GM information about all the samples. The Venn diagram (Figure 2) which was plotted according to the OTUs cluster analysis showed that there were 1395 common OTUs in all five groups.

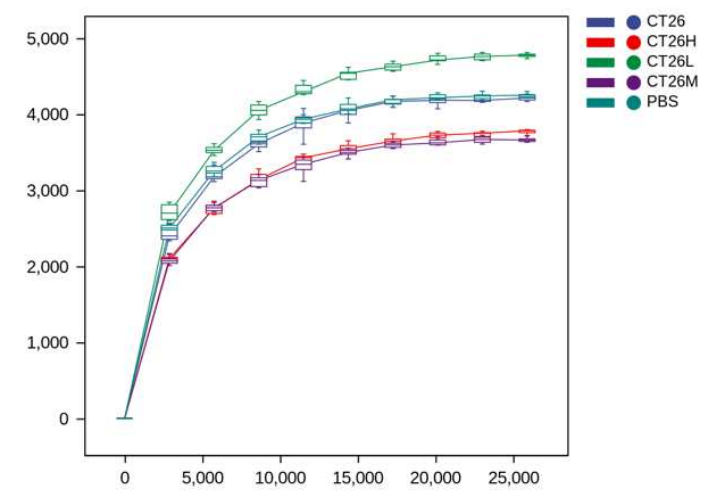

Figure 1 The rarefaction curves of each group based on Chao1

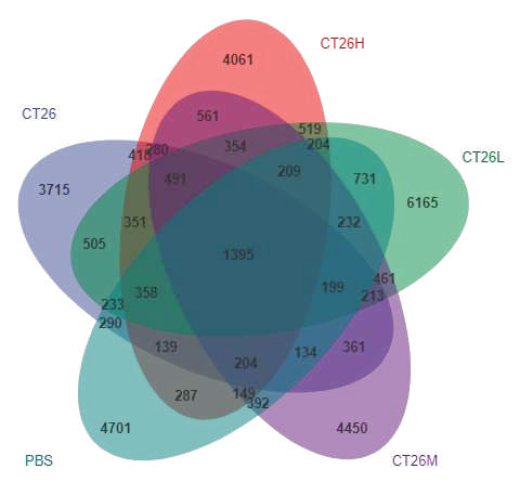

Figure 2 Venn diagram of OTUs in each group

The results of statistical analysis showed a significant difference in GM between the Tumor-bearing mice and healthy mice. The principal co-ordinates analysis (PCA) reflected the similarity in species abundance composition of two samples in the corresponding dimensions by analyzing the projection distance of the samples on the coordinate axis. As for CT26 group and PBS group, the PCA diagram (Figure 3(a)) showed that the species abundance composition of the two groups can be classified obviously. The Rank Abundance Curve consists of broken lines, and each broken line represents a group. The length of the broken line on the horizontal axis reflects the number of OTU in the specific abundance. The smoother the broken line is, the smaller difference of the OTU diversity in the community is. As for CT26 group and PBS group, the abundance grade curve (Figure 3(b)) illustrated that the Healthy Group (PBS) has higher community evenness than Tumor-bearing group (CT26). The alpha diversity analysis (Figure 3(c)) showed that there were no significant differences in Chao1, Good's coverage, Shannon, Simpson and Observed species indices between CT26 group and PBS group. However, beta diversity analysis using both unweighted and weighted UniFrac distances indicated that PBS group had higher dissimilarities among gut microbial communities than CT26 group (Table 1,2). 


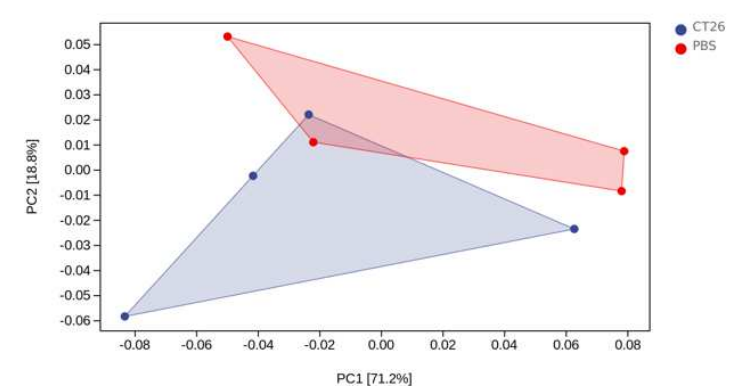

(a)

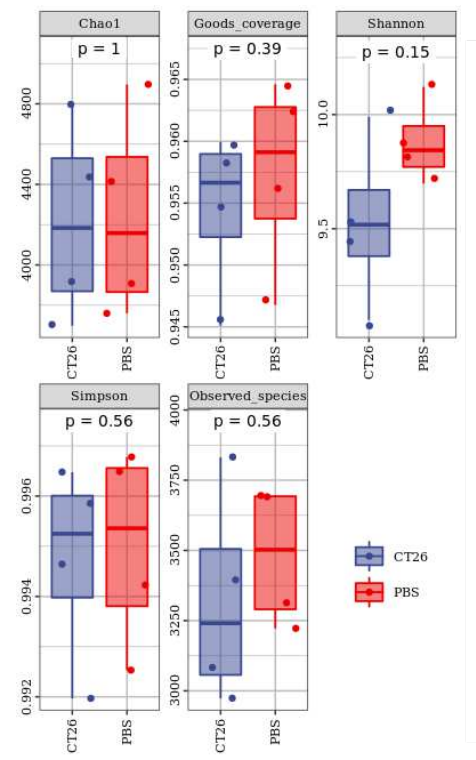

(c)

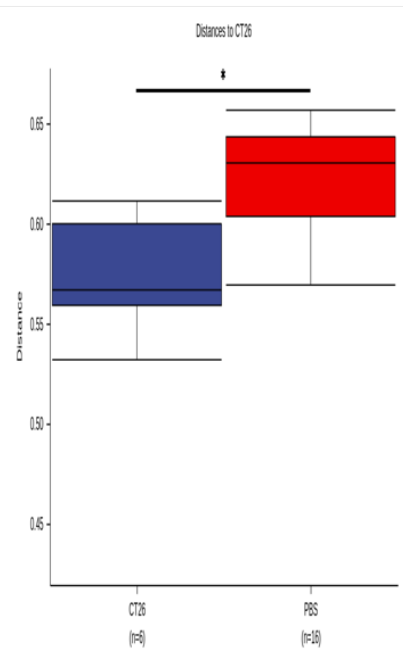

(d)

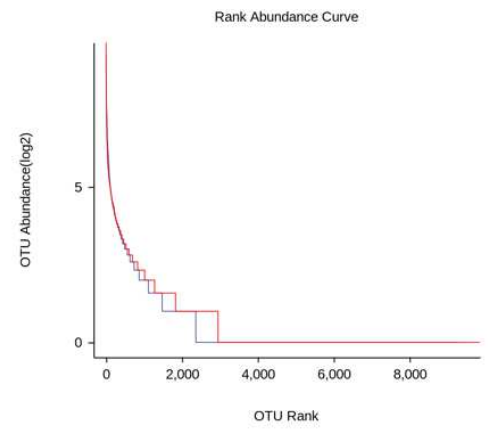

(b)

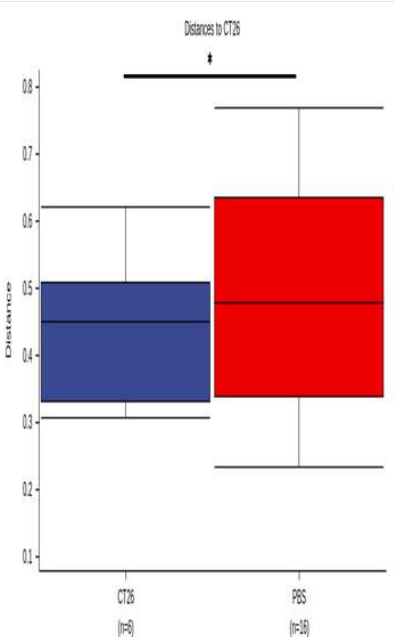

(e)

Figure 3 (a) The PCA diagram of CT26 group and PBS group. (b) The abundance grade curve of CT26 group and PBS group. (c) The differences in Chao1, Good's coverage, Shannon, Simpson and Observed species indices between CT26 group and PBS group. (d) The differences in unweighted UniFrac distance between CT26 group and PBS group. (e) The differences in weighted UniFrac distance between CT26 group and PBS group.

The taxonomic summary figure (Figure4(b)) showed that the composition of GM in the two groups (CT26 group and PBS group) was mainly characterized by the Firmicutes, Bacteroidetes, Proteobacteria, TM7 and Deferribacteres. A marked difference between the two groups existed on the distribution of phyla. The abundance of Firmicutes and Deferribacteres in PBS group was higher than that in CT26 group, while the abundance of Bacteroidetes and Proteobacteria was lower. As showed in Figure4(b), species composition in two groups at genus level was quite different as well. Species with high expression in CT26 group were Lactobacillus, Alistipes, 
Parabacteroides, [Prevotella], Adlercreutzia, Odoribacter, Bacteroides, Desulfovibrio, Turicibacter, Coprococcus, Helicobacter. And species with low expression in CT26 group were Rikenella, Oscillospira, Roseburia, Ruminococcus, Flexispira, [Ruminococcus], Butyricicoccus, Mucispirillum, Prevotella.

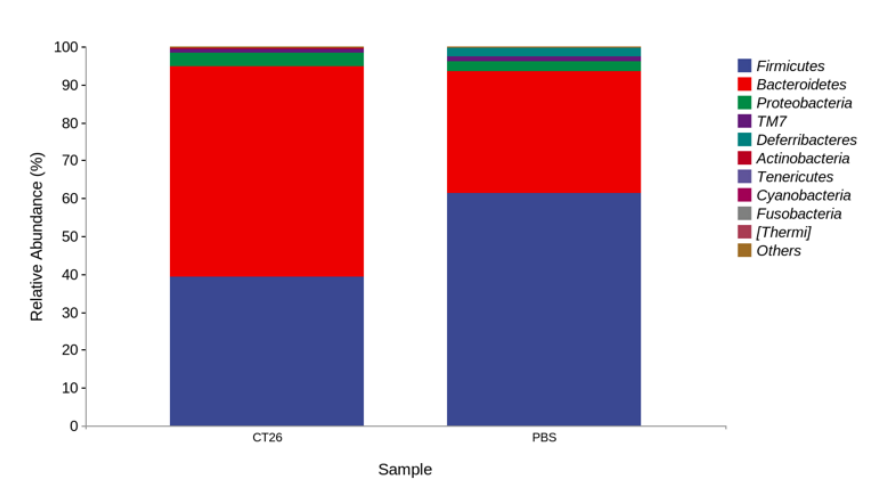

(a)

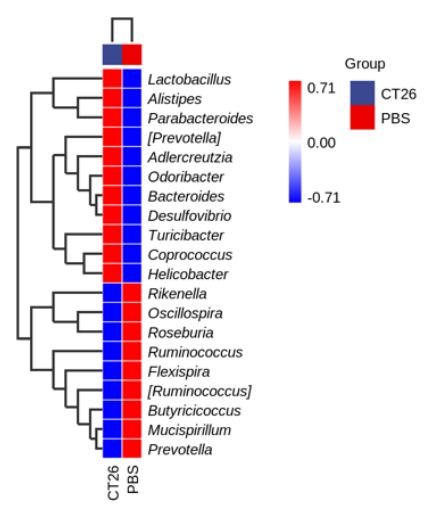

(b)

Figure4 (a) Taxonomic summary of the gut microbiota at phylum level. (b) The heat map of species composition of the gut microbiota in CT26 group and PBS group at genus level.

\subsection{The present of tumor affected the absorption of FFCT}

A high dose of FFCT $(2.6 \mathrm{mg} / \mathrm{g})$ was used both in CT26 group (to be CT26-CM group) and PBS group (to be CM group) to compare the serum metabolism between the two groups. In the PCA plots (Figure 5(a)), the quality control (QC) samples were closely gathered in the center of the scoring chat, showing the good stability of the instrument detection in this experiment. In the Metabolites Intensity Distribution box plot (Figure 5(b)), the median line of each group was on a horizontal line, indicating that the samples were relatively stable. And the dots below represented the degree of dispersion, showing a relatively low dispersion in CM group.

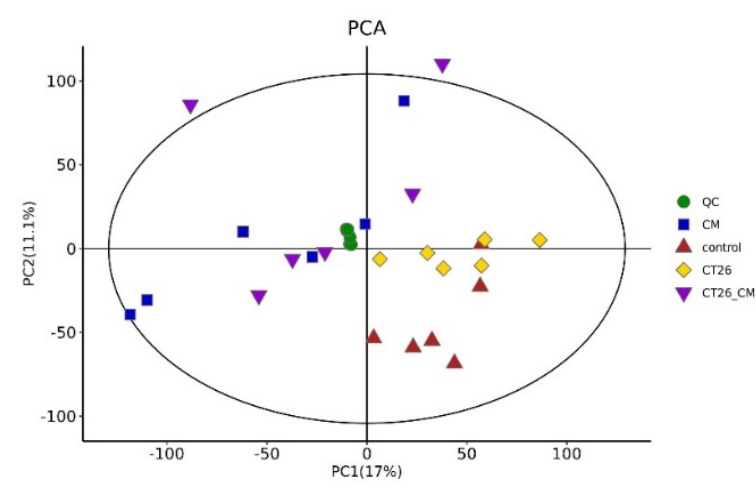

(a)

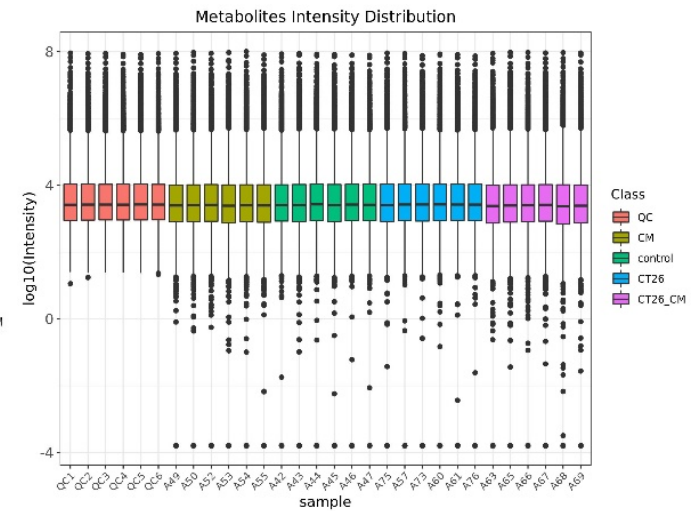

(b)

Figure 5 Quality control chart. (a) PCA score plot of all samples. (b) Metabolites Intensity 
Distribution box plot of all samples.

The OPLS-DA plot can reflect the variability between groups and within groups, and can observe the general distribution trend among samples. As Figure 6 showed, samples of the two groups were separated clearly, which meant that the repeatability within each group was good, the difference between the two groups was large and the data characteristics between different groups were significantly different.

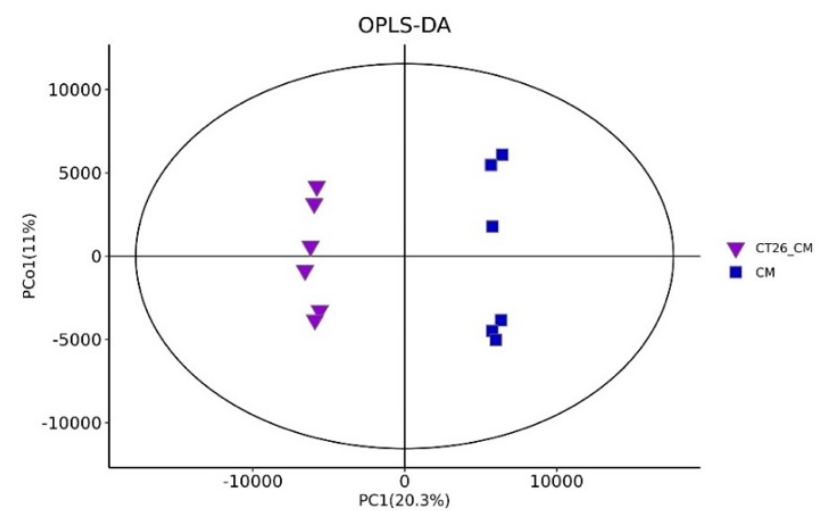

Figure 6 OPLS-DA score plot of tumor-bearing group treating by FFCT (CT26-CM group) and FFCT-treating group (CM group). R2=(0.0,0.981), Q2=(0.0,-0.159).

There were 225 different metabolites between CT26-CM group and CM group. In order to show the relationship between the two groups and the expression differences in metabolites among different samples more intuitively, the hierarchical clustering heatmap was used to visualize the different potential biomarkers between these two groups. Top 50 difference metabolites and KEGG pathways enriched by significantly different metabolites were identified as listed in Figure 7. Combined with network retrieval, serum metabolites that related to FFCT like Citric acid, ( \pm ) 12-HEPE, Cycloartanyl ferulate, 5Z,8Z,14Z-Eicosatrien-11-ynoic acid , $( \pm) 12$ - HETE, PE(0:0/22:6(4Z,7Z,10Z,13Z,16Z,19Z)), PE(16:0/0:0), LysoPE(18:0/0:0), 1-(2-methoxy-13-methyl-6Z-tetradecenyl)-sn-glycero-3-phosphoethanolamine , Oseltamivir phosphate and 1-(2-methoxy-6Z-heptadecenyl)-sn-glycero-3-phosphoethanolamine were much more in CM group than in CT26-CM group, indicating that the present of tumor could affect the metabolism of FFCT(Figure 7(a)). As Figure 7(b) showed, these differential metabolites of FFCT involved in multiple pathways including the Alanine, aspartate and glutamate metabolism, Central carbon metabolism in cancer, Biosynthesis of amino acids, et al. 


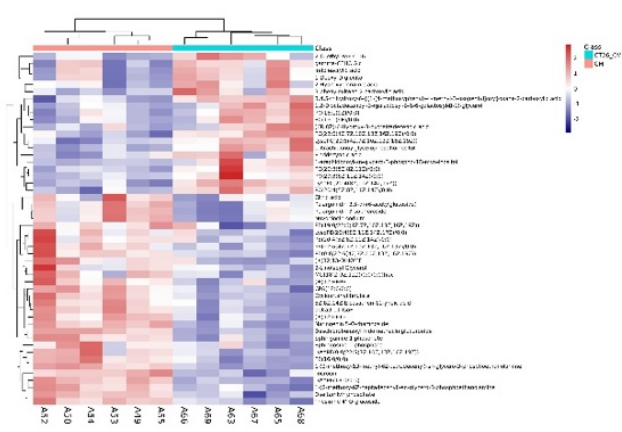

(a)

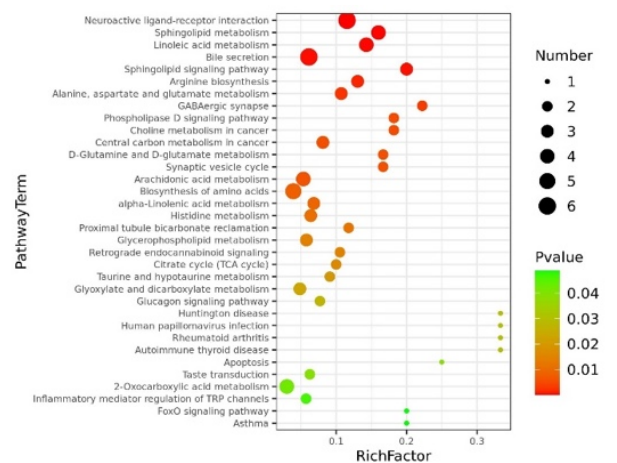

(b)

Figure 7 The hierarchical clustering heatmaps. (a) The hierarchical clustering heatmap of different metabolites in CT26-CM group and CM group. (b) KEGG pathways enriched by significantly different metabolites between CT26-CM group and CM group $(p<0.05)$.

\subsection{FFCT ameliorated the GM in tumor-bearing mice}

As the above results shown, GM and blood metabolism of FFCT between tumor-bearing group and healthy group were quite different. The dysbiosis of GM in tumor-bearing mice was corresponding to less serum metabolites that related to FFCT. Next, 16S rRNA gene sequencing was used to detect the effect of different doses of FFCT on the GM of tumor-bearing mice. As expected, compared with the CRC tumor-bearing group (CT26), FFCT-treating groups (CT26-L, CT26-M, CT26-H) had different composition of GM. The PCA diagram (Figure 8(a)) showed that the species abundance composition of FFCT-treating groups was different from that in CT26 group, and could be classified obviously. The abundance grade curve (Figure 8(b)) showed that the FFCT-treating groups had higher community evenness than CT26 group. The alpha diversity analysis (Figure 8(c)) showed that there were no significant differences in Chao1, Good's coverage, Shannon, Simpson and Observed species indices between CT26 group and FFCT-treating group. However, beta diversity analysis using both unweighted and weighted UniFrac distances indicated that the medium dose FFCT-treating group had higher dissimilarities among gut microbial communities than CT26 group (Table 3,4).
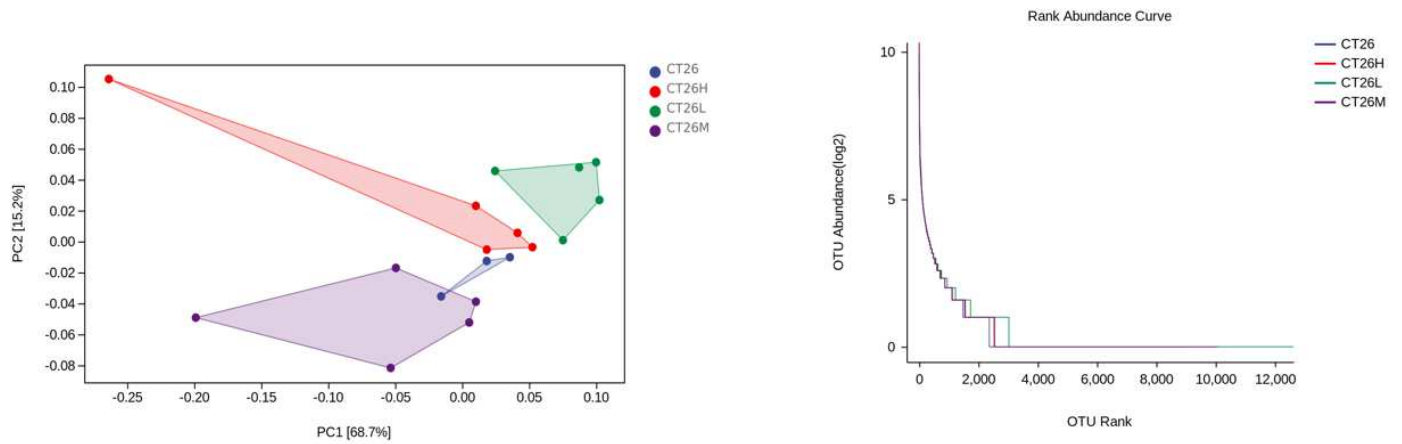
(a)

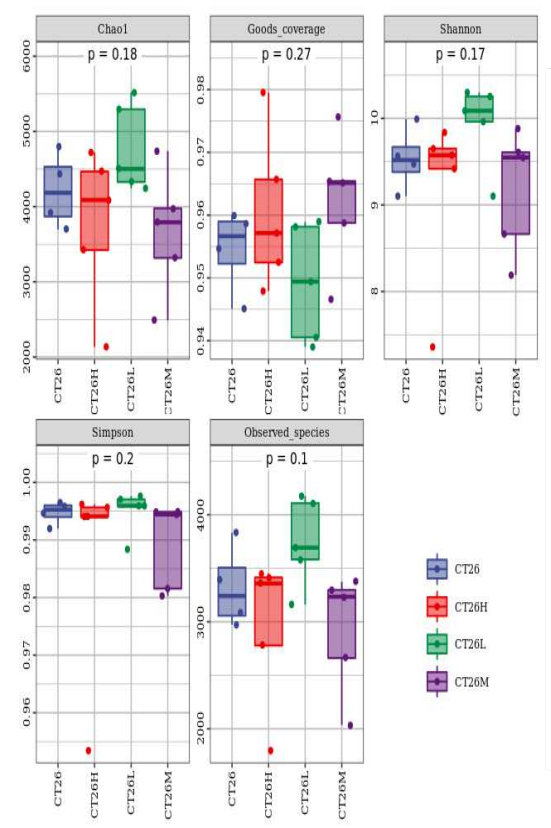

(c)

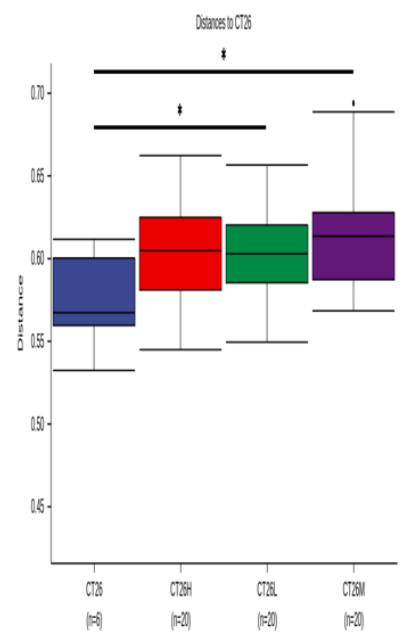

(d) (b)

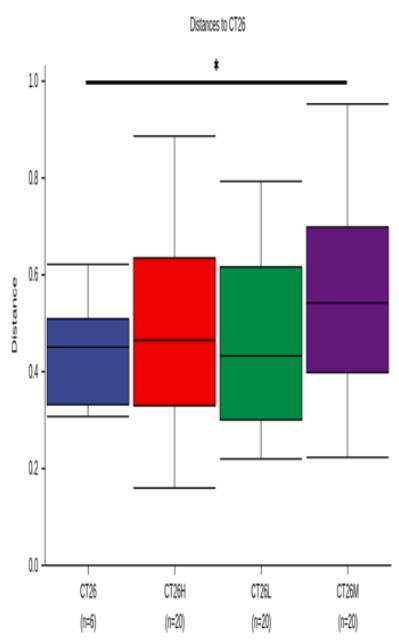

(e)

Figure 8 (a) The PCA diagram of CT26 group and FFCT-treating groups. (b) The abundance grade curve of CT26 group and FFCT-treating groups. (c) The differences in Chao1, Good's coverage, Shannon, Simpson and Observed species indices between CT26 group and FFCT-treating groups. (d) The differences in unweighted UniFrac distance between CT26 group and FFCT-treating groups. (e) The differences in weighted UniFrac distance between CT26 group and FFCT-treating groups.

The taxonomic summary figure (Figure9(a)) showed that the composition of GM in the four groups was mainly characterized by the Firmicutes, Bacteroidetes, Proteobacteria, TM7 and Deferribacteres. In the distribution of phyla, FFCT-treating groups had higher abundance of Firmicutes than tumor-bearing group, which showed a similar trend with Healthy group. And with the increase in FFCT concentration, the abundance of Firmicutes got decreased. On the contrary, FFCT-treating groups had lower abundance of Bacteroidetes than tumor-bearing group, which also showed a similar trend with Healthy group. The CT26-M group had the least abundance of Bacteroidetes and the most abundance of Proteobacteria and Actinobacteria.

As showed in Figure9(b), species with high expression in CT26 group but low expression in FFCT-treating groups were as follows: Odoribacter, [Prevotella], Prevotella, Coprococcus, Parabacteroides. On the contrary, species like Roseburia, Turicibacter and Flexispira in CT26 group were much less than in FFCT-treating groups. Among these species, Roseburia and Turicibacter increased with the increase of FFCT concentration. 


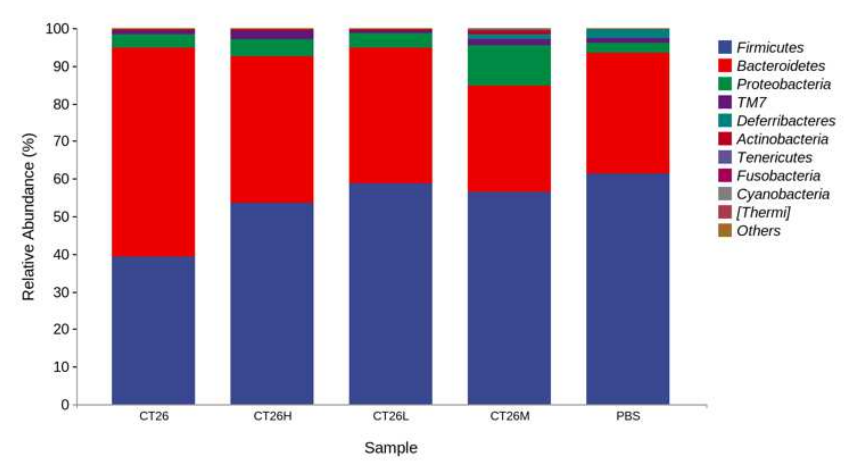

(a)

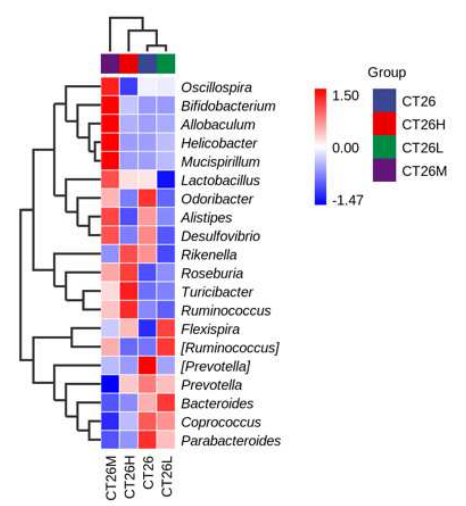

(b)

Figure9 (a) Taxonomic summary of the gut microbiota at phylum level. (b) The heat map of species composition of the gut microbiota in CT26 group and FFCT-treating groups at genus level.

\section{Discussion}

In the adjuvant treatment of tumor, Chinese medicine has become an essential part due to its multi-target effect and low toxicity. However, the mechanism of the curative effect is complicated. Studies showed that emodin could induce the cancer cell apoptosis via endoplasmic reticulum stress-dependent events, which produced reactive oxygen species (ROS) and regulated the signaling pathways ${ }^{[23]}$. Qinrui $\operatorname{Han}^{[24]}$ et al., showed that bufarenogin induced intrinsic apoptosis through the cooperation of Bax and adenine-nucleotide translocator. Xiao-Qin $\mathrm{Zhu}^{[25]}$ et al., treated CRC HCT-116 xenograft mouse model with Qingjie Fuzheng Granules and found that this compound suppressed tumor cell proliferation through inhibiting SHh pathway. Yang $\mathrm{Li}^{[26]}$ et al., did research on Gegen Qinlian decoction. They tested the immune cells, the cytokines and the intestinal mucosa tight protein. Combining with the results of gut microbiota (GM), they concluded that Gegen Qinlian decoction could enhance immunity and protect intestinal barrier function by decreasing the relative abundance of Megamonas and Veillonella, and increasing the relative abundance of Bacteroides, Akkermansia and Prevotella. As Yang's study shown, GM had become a considerable factor in the development and treatment of CRC.

Jiyoung $A n^{[27]}$ et al., tested the community of GM in a study of 47 CRC subjects and 94 control subjects. From such a clinical research, they found that CRC case subjects decreased overall microbial community diversity, showing the potentially modifiable nature of GM and the difference of GM between CRC patients and healthy people. GM is an important part of intestinal mucosal barrier and intestinal immunity. GM can influence physiological and pathological processes throughout the whole body, including the bioavailability and meta-ballism of 
macronutrients and micronutrients as well as metabolites. The vast majority of Chinese medicine are absorbed through intestinal tract to achieve the therapeutic effect, so there is the hypothesis that GM could affect the absorption and metabolism of Chinese medicine.

In this study, we chose Fufangchangtai (FFCT) formula as the model prescription. FFCT decoction was created by the famous Chinese Medicine Practitioner Professor Jiege Huo through summing up decades of experience in the CRC therapy. The previous multicenter, randomized, double-blind clinical trial had confirmed that FFCT had a certain effect on improving the life quality, enhancing immune function and prolonging the median survival time of CRC patients. However, the mechanism of its anti-tumor effect is unclear, that's the reason for choosing FFCT as the research object.

Instead of AOM/DSS induction mice, the subcutaneous transplantation mice were used as the CRC tumor-bearing mice model. The AOM/DSS induction mice model is induced by administration through gastrointestinal tract, which not only affects the experimental results of GM but also has the disadvantages of long modeling cycle, unstable effect and high mortality. The subcutaneous transplantation mice model is more intuitive and makes the experimental results stable and reliable.

In order to verify the difference in GM among different groups of mice, the GM in fecal of CRC mice and healthy mice was detected. The results showed that the abundance of Firmicutes decreased and Bacteroidetes increased in tumor-bearing mice, which were consistent with findings from clinical analysis of CRC patients ${ }^{[28]}$. As our study shown, Coprococcus, Helicobacter, Desulfovibrio, et al., had high expression in CT26 group. Coprococcus, which is related to butyrate production ${ }^{[29]}$ and the metabolism of SCFAs ${ }^{[30]}$, increased in tumor-bearing mice. Sarah Vascellari's study proposed that the high expression of Coprococcus was associated with the development of gut inflammatory environment and gastrointestinal dysfunctions ${ }^{[31]}$, which were pathological factors of CRC. Helicobacter, especially helicobacter_hepaticus, can lead to an increase in oxidative phosphorylation that may increase DNA-damaging free radicals ${ }^{[32]}$. Verena Friedrich $^{[33]}$ colonized the spontaneous fatal colitis transgenic mouse modelled with helicobacter_hepaticus and found the disease developed rapidly, showing that this kind of bacteria was a disease driver in the model of colitis and was not conducive to maintain intestinal homeostasis. Desulfovibrio is considered as the virulent bacteria which plays a role in destroying colonic mucosa and inducing intestinal inflammation and bacterial translocation ${ }^{[34]}$. Since the significant difference in GM between CRC tumor-bearing mice (CT26 group) and healthy mice (PBS group), the further study was allowed to be carried out in the tumor-bearing model mice.

We detected the blood metabolism of tumor-bearing mice and healthy mice after 
administration of FFCT to explore whether the dysbiosis of GM under the burden of tumor had an impact on serum metabolism that related to TCM. Metabolites that related to FFCT like Citric acid, ( \pm ) 12-HEPE, Cycloartanyl ferulate, $\mathrm{PE}(16: 0 / 0: 0)$, et al., were much more in CM group than in CT26-CM group. Citric acid is the product of tricarboxylic acid cycle, helping transform food into usable energy. Studies ${ }^{[35-37]}$ showed that citric acid could enhance the nutrient absorption including enhancing bioavailability of minerals and the absorption of zinc supplements. Moreover, citric was identified to have high correlation coefficients with proangiogenic activity ${ }^{[38]} .( \pm)$ 12-HEPE is a kind of circulating hydroxy polyunsaturated fatty acids ${ }^{[39]}$, and is recently identified as BAT (brown adipose tissue) -derived circulating factors (BATokines), which plays a critical important role in regulating the systemic lipid pool that related to energy dissipation ${ }^{[40]}$. Cycloartenyl ferulate is a component of gamma-oryzanol, which was found to have the function of capturing $\operatorname{IgE}$ and attenuating the allergic reaction ${ }^{[4]}$. Islam et al., investigated the effect of $\gamma$-oryzanol, Cycloartenyl ferulate and ferulic acid on a model of colitis in mice. The research found that Cycloartenyl ferulate had strong antioxidant effects so to inhibit NF-kB activity and then to ameliorate colonic inflammation ${ }^{[42]}$, showing the potential for treating or preventing the gastrointestinal inflammatory diseases. The above three main different metabolites indicated that FFCT produced a marked effect on providing nutrition and energy and regulating microenvironment. The results suggested that there were more serum metabolites that related to FFCT in healthy group. Combing with the results of GM, the conclusion could be summarized that a relatively healthy intestinal flora and microenvironment was conductive to the absorption and metabolism of drugs into the blood, so as to play a better therapeutic effect.

Further analysis of metabolic pathways showed that the therapeutic effect of FFCT might be predominantly relevant to the ability of regulating Alanine, aspartate and glutamate metabolism, Central carbon metabolism in cancer, Biosynthesis of amino acids. Alanine, aspartate and glutamate metabolism involves in the synthesis of arginine and purine, the interconversion of $\mathrm{C} 3$ and $\mathrm{C} 4$, and the activity of the DcuS-DcuR two-component system ${ }^{[43]}$. Ryosei Sakai et al., investigated permeability and metabolism of dietary glutamate in Caco-2 intestinal epithelial cell layer model and found that the intestinal epithelial cell monolayer could utilize dietary glutamate to maintain glutamate homeostasis in the body ${ }^{[44]}$. Central carbon metabolism traditionally includes glycolysis pathway (EMP), pentose phosphate pathway (PPP) and tricarboxylic acid cycle (TCA), and it is the main source of energy required by organisms and provides precursors to other metabolism in the body. As amino acids are the basic units of protein molecules and give play to activating immune system in organisms such as immunoglobulin synthesis, lymphocyte quantity and phagocyte function ${ }^{[45]}$. These results indicated that FFCT could keep the balance of 
internal environment through intestinal absorption and metabolism so as to involve in the synthesis of substance and supplement of energy in serum metabolism.

At the same time, FFCT was administrated to CRC tumor-bearing mice to observe the alteration of GM under the intervention of TCM. It was also found that there were significant differences in the composition of GM in tumor-bearing mice before and after FFCT administration. Many evidences demonstrated that Firmicutes was a phylum with documented anti-tumorigenic effects $^{[46]}$. Bacteroidetes on the contrary, could drive DNA damages in colon epithelial cells as a potential "driver" of $\mathrm{CRC}^{[47]}$. As the abundance of Firmicutes was higher and the abundance of Bacteroidetes was lower in FFCT-treating groups, and Firmicutes/Bacteroidetes was generally regarded as significant relevance in signaling $\mathrm{GM}$ status ${ }^{[48]}$, the results indicated that the imbalance of GM in tumor-bearing mice was corrected. The intervention of FFCT increased the abundance of Turicibacter and Roseburia, and as the FFCT concentration increased, the abundance of these two species of phylum increased. Turicibacter is involved in fermentation metabolism and its main metabolite is lactic acid, which has the function of regulating muscle and anti fatigue. Roseburia can ferment a variety of carbohydrates, increase the content of butyric acid in the intestine, and has the effect of preventing or treating obesity related diseases. Studies showed that Turicibacter and Roseburia had a negative association with intestinal dystrophy ${ }^{[49]}$. Combining with the research results, the conclusion could be drawn that FFCT changed the structure of GM and maintained the enteral nutrition. However, the specific intestinal protection mechanism remains to be further studied.

\section{Conclusion}

It was pointed out that the GM should be concerned during the therapy of FFCT. The more healthier intestinal microenvironment was conductive to the better efficacy of FFCT. On the contrary, a dysbiosis GM under CRC burden blocked the absorption and metabolism of FFCT. In addition, FFCT could correct the imbalance GM of CRC individual. This research provided a new viewpoint to study the therapeutic effect of traditional Chinese medicine from the perspective of combining gut microbiota and serum metabolism, and also provided a basis for improving the GM status of CRC patients in clinical treatment, aiming to achieve better curative effect.

\section{Declarations}

\section{Funding}

This research was funded by Jiangsu Postgraduate Research and Practice Innovation Program (Grant Number SJCX20_0519): Research on anti colorectal cancer mechanism of Fufangchangtai based on gut microbiota. 


\section{Author information}

Affiliations

Nanjing University of Chinese Medicine, Nanjing, Jiangsu 210046, China

Mengmeng Cai, Ya Xiao, Jialin Gu, Yuzhu Ma

Department of Oncology, Affiliated Hospital of Integrated Traditional Chinese and Western Medicine, Nanjing University of Chinese Medicine, Nanjing, Jiangsu 210028, China

Mengmeng Cai, Ya Xiao, Jialin Gu, Yuzhu Ma, Jiege Huo

Shanghai Jiao Tong University School of Agriculture and Biology, Shanghai 201100, China

Zhibing Lin, Xiaoyu Wang

Department of Oncology, Jiangsu Province Academy of Traditional Chinese Medicine, Nanjing, Jiangsu 210028, China

Ya Xiao, Jiege Huo

South China Agricultural University, Guangzhou, Guangdong 510642, China

Jinmiao Lu, Shilan Zhu, Xiaoyu Chen

Shanghai Veterinary Research Institute, Chinese academy of Agricultural Sciences, Shanghai 201100, China

Mengmeng Cai, Jinmiao Lu, Shilan Zhu, Xiaoyu Chen, Zhaoguo Chen

\section{Contributions}

Mengmeng Cai performed laboratory work and wrote first draft. Zhibing Lin developed the concept, managed samples collection, and supervised laboratory work and data analysis. Ya Xiao performed revision and contributed to writing manuscript. Jinmiao Lu, Xiaoyu Wang, Shilan Zhu and Xiaoyu Chen performed laboratory work. Jialin Gu and Yuzhu Ma managed samples collection. Jiege Huo and Zhaoguo Chen supervised, reviewed and edited the manuscript. All authors read and approved the final manuscript.

\section{Corresponding authors}

Correspondence to Jiege Huo and Zhaoguo Chen. 


\section{Ethics declarations}

Ethics approval and consent to participate

The study was approved by the Laboratory animal welfare and Ethics Management Committee of Shanghai Veterinary Research Institute (ethics number: SHVRI-SZ-20200420-01,

SHVRI-SZ-20200720-01).

\section{Consent for publication}

All the authors have read the paper before submission and consent submuission.

\section{Competing interests}

The authors declare that there are no conflicts of interest regarding the publication of this paper.

\section{Data availability}

The data used to support the findings of this study are available from the corresponding author upon request.

\section{Acknowledgements}

For the completion of my thesis, first, I wish to express my deepest gratitude to my supervisor, Prof. Jiege Huo and Prof. Zhaoguo chen, who has given me the most valuable suggestions and advice, and made necessary corrections. Then I am greatly grateful to Dr. Zhibing Lin and Dr. Jinmiao $\mathrm{Lu}$ for their help in my laboratory work and data analysis.

I am also deeply indebted to all the other teachers and colleagues for their direct and indirect help to me.

Special thanks should go to my parents and friends for their continuous support and encouragement.

\section{References}

1. Siegel RL, Miller KD, Jemal A: Cancer statistics, 2020. CA Cancer J Clin 2020, 70(1):7-30.

2. Milosevic I, Vujovic A, Barac A, Djelic M, Korac M, Radovanovic Spurnic A, Gmizic I, Stevanovic O, Djordjevic $\mathrm{V}$, Lekic $\mathrm{N}$ et al: Gut-Liver Axis, Gut Microbiota, and Its Modulation in the Management of Liver Diseases: A Review of the Literature. Int J Mol Sci 2019, 20(2).

3. Eckburg PB, Bik EM, Bernstein CN, Purdom E, Dethlefsen L, Sargent M, Gill SR, Nelson KE, Relman DA: Diversity of the human intestinal microbial flora. Science 2005, 308(5728):1635-1638.

4. Ramakrishna BS: Role of the gut microbiota in human nutrition and metabolism. $J$ Gastroenterol Hepato/ 2013, 28 Suppl 4:9-17.

5. Castellanos JG, Woo V, Viladomiu M, Putzel G, Lima S, Diehl GE, Marderstein AR, Gandara J, Perez AR, Withers DR et al: Microbiota-Induced TNF-like Ligand 1A Drives Group 3 Innate Lymphoid Cell-Mediated Barrier Protection and Intestinal T Cell 
Activation during Colitis. Immunity 2018, 49(6):1077-1089.e1075.

6. Sobhani I, Tap J, Roudot-Thoraval F, Roperch JP, Letulle S, Langella P, Corthier G, Tran Van Nhieu J, Furet JP: Microbial dysbiosis in colorectal cancer (CRC) patients. PloS one 2011, 6(1):e16393.

7. Gagniere J, Raisch J, Veziant J, Barnich N, Bonnet R, Buc E, Bringer MA, Pezet D, Bonnet M: Gut microbiota imbalance and colorectal cancer. World journal of gastroenterology 2016, 22(2):501-518.

8. Niccolai E, Russo E, Baldi S, Ricci F, Nannini G, Pedone M, Stingo FC, Taddei A, Ringressi MN, Bechi P et al: Significant and Conflicting Correlation of IL-9 With Prevotella and Bacteroides in Human Colorectal Cancer. Front Immuno/ 2020, 11:573158.

9. Fidelle M, Yonekura S, Picard M, Cogdill A, Hollebecque A, Roberti MP, Zitvogel L: Resolving the Paradox of Colon Cancer Through the Integration of Genetics, Immunology, and the Microbiota. Front Immuno/2020, 11:600886.

10. Zhang Z, Shao S, Zhang Y, Jia R, Hu X, Liu H, Sun M, Zhang B, Li Q, Wang Y: Xiaoyaosan slows cancer progression and ameliorates gut dysbiosis in mice with chronic restraint stress and colorectal cancer xenografts. Biomedicine \& pharmacotherapy = Biomedecine \& pharmacotherapie 2020, 132:110916.

11. El Joumaa MM, Taleb RI, Rizk S, Borjac JM: Protective effect of Matricaria chamomilla extract against 1,2-dimethylhydrazine-induced colorectal cancer in mice. J Complement Integr Med 2020, 17(3).

12. LI LC: Randomized, double-blind controlled study of Fufangchangtai granule in the treatment of colon cancer. Doctoral Dissertation. Nanjing University of Chinese Medicine; 2019.

13. $\mathrm{Xu}$ J, Chen HB, Li SL: Understanding the Molecular Mechanisms of the Interplay Between Herbal Medicines and Gut Microbiota. Medicinal research reviews 2017, 37(5):1140-1185.

14. Yu YN, Yu TC, Zhao HJ, Sun TT, Chen HM, Chen HY, An HF, Weng YR, Yu J, Li M et at. Berberine may rescue Fusobacterium nucleatum-induced colorectal tumorigenesis by modulating the tumor microenvironment. Oncotarget 2015, 6(31):32013-32026.

15. Sui H, Zhang L, Gu K, Chai N, Ji Q, Zhou L, Wang Y, Ren J, Yang L, Zhang B et al: YYFZBJS ameliorates colorectal cancer progression in $\operatorname{Apc}(\mathrm{Min} /+)$ mice by remodeling gut microbiota and inhibiting regulatory T-cell generation. Cell Commun Signal 2020, 18(1):113.

16. Wang JH: Mechanisms of FFCT on inducing autophagy of colon cancer cells and promoting polarization of tumor-associated macrophages. Doctoral Dissertation. Nangjing Medical University; 2018.

17. Xu SY, Bian RL, Chen X: Pharmacology experimental methodology (2nd Edition). Chin Pharmacol Bull 1992(01):19.

18. Church DL, Cerutti L, Gürtler A, Griener T, Zelazny A, Emler S: Performance and Application of 16S rRNA Gene Cycle Sequencing for Routine Identification of Bacteria in the Clinical Microbiology Laboratory. Clin Microbiol Rev 2020, 33(4).

19. Tian Y, Wang Z, Liu X, Duan J, Feng G, Yin Y, Gu J, Chen Z, Gao S, Bai H et al: Prediction of Chemotherapeutic Efficacy in Non-Small Cell Lung Cancer by Serum Metabolomic Profiling. Clin Cancer Res 2018, 24(9):2100-2109. 
20. Scanlan PD, Shanahan F, Clune Y, Collins JK, O'Sullivan GC, O'Riordan M, Holmes E, Wang $Y$, Marchesi JR: Culture-independent analysis of the gut microbiota in colorectal cancer and polyposis. Environ Microbio/ 2008, 10(3):789-798.

21. Marchesi JR, Dutilh BE, Hall N, Peters WH, Roelofs R, Boleij A, Tjalsma H: Towards the human colorectal cancer microbiome. PloS one 2011, 6(5):e20447.

22. Wu S, Rhee KJ, Albesiano E, Rabizadeh S, Wu X, Yen HR, Huso DL, Brancati FL, Wick E, McAllister $F$ et at: A human colonic commensal promotes colon tumorigenesis via activation of $\mathrm{T}$ helper type $17 \mathrm{~T}$ cell responses. Nature medicine 2009, 15(9):1016-1022.

23. Zhang Y, Pu W, Bousquenaud M, Cattin S, Zaric J, Sun LK, Rüegg C: Emodin Inhibits Inflammation, Carcinogenesis, and Cancer Progression in the AOM/DSS Model of Colitis-Associated Intestinal Tumorigenesis. Front Onco/ 2020, 10:564674.

24. Han Q, Zhang C, Zhang Y, Li Y, Wu L, Sun X: Bufarenogin induces intrinsic apoptosis via Bax and ANT cooperation. Pharmacol Res Perspect 2021, 9(1):e00694.

25. Zhu XQ, Yang H, Lin MH, Shang HX, Peng J, Chen WJ, Chen XZ, Lin JM: Qingjie Fuzheng Granules regulates cancer cell proliferation, apoptosis and tumor angiogenesis in colorectal cancer xenograft mice via Sonic Hedgehog pathway. J Gastrointest Oncol 2020, 11(6):1123-1134.

26. Li Y, Li ZX, Xie CY, Fan J, Lv J, Xu XJ, Lv J, Kuai WT, Jia YT: Gegen Qinlian decoction enhances immunity and protects intestinal barrier function in colorectal cancer patients via gut microbiota. World journal of gastroenterology 2020, 26(48):7633-7651.

27. Ahn J, Sinha R, Pei Z, Dominianni C, Wu J, Shi J, Goedert JJ, Hayes RB, Yang L: Human gut microbiome and risk for colorectal cancer. Journal of the National Cancer Institute 2013, 105(24):1907-1911.

28. Abreu MT, Peek RM: Gastrointestinal Malignancy and the Microbiome. Gastroenterology 2014, 146(6):1534-1546.e1533.

29. Lin L, Trabi EB, Xie F, Mao S: Comparison of the fermentation and bacterial community in the colon of Hu sheep fed a low-grain, non-pelleted, or pelleted high-grain diet. App/ Microbiol Biotechno/ 2021.

30. Liu C, Du P, Cheng Y, Guo Y, Hu B, Yao W, Zhu X, Qian H: Study on fecal fermentation characteristics of aloe polysaccharides in vitro and their predictive modeling. Carbohydrate polymers 2021, 256:117571.

31. Vascellari S, Melis M, Palmas V, Pisanu S, Serra A, Perra D, Santoru ML, Oppo V, Cusano R, Uva P et at: Clinical Phenotypes of Parkinson's Disease Associate with Distinct Gut Microbiota and Metabolome Enterotypes. Biomolecules 2021, 11(2).

32. Wang Z, Fu H, Zhou Y, Yan M, Chen D, Yang M, Xiao S, Chen C, Huang L: Identification of the gut microbiota biomarkers associated with heat cycle and failure to enter oestrus in gilts. Microb Biotechno/ 2020.

33. Friedrich V, Forné I, Matzek D, Ring D, Popper B, Jochum L, Spriewald S, Straub T, Imhof $A$, Krug $A$ et al: Helicobacter hepaticus is required for immune targeting of bacterial heat shock protein 60 and fatal colitis in mice. Gut Microbes 2021, 13(1):1-20.

34. Yan S, Tian S, Meng Z, Teng M, Sun W, Jia M, Zhou Z, Bi S, Zhu W: Exposure to nitenpyram during pregnancy causes colonic mucosal damage and non-alcoholic 
steatohepatitis in mouse offspring: The role of gut microbiota. Environ Pollut 2021, 271:116306.

35. Lacour B, Tardivel S, Drüeke T: Stimulation by citric acid of calcium and phosphorus bioavailability in rats fed a calcium-rich diet. Miner Electrolyte Metab 1997, 23(2):79-87.

36. Teucher B, Olivares M, Cori $\mathrm{H}$ : Enhancers of iron absorption: ascorbic acid and other organic acids. Int J Vitam Nutr Res 2004, 74(6):403-419.

37. Wegmüller R, Tay F, Zeder C, Brnic M, Hurrell RF: Zinc absorption by young adults from supplemental zinc citrate is comparable with that from zinc gluconate and higher than from zinc oxide. J Nutr 2014, 144(2):132-136.

38. Liu M, Zhao L, Han L, Li H, Shi Y, Cui J, Wang C, Xu L, Zhong L: Discovery and identification of proangiogenic chemical markers from Gastrodiae Rhizoma based on zebrafish model and metabolomics approach. Phytochem Anal 2020, 31(6):835-845.

39. Wang T, Li H, Han Y, Wang Y, Gong J, Gao K, Li W, Zhang H, Wang J, Qiu X et al. A rapid and high-throughput approach to quantify non-esterified oxylipins for epidemiological studies using online SPE-LC-MS/MS. Anal Bioanal Chem 2020, 412(28):7989-8001.

40. Kulterer OC, Niederstaetter L, Herz CT, Haug AR, Bileck A, Pils D, Kautzky-Willer A, Gerner C, Kiefer FW: The Presence of Active Brown Adipose Tissue Determines Cold-Induced Energy Expenditure and Oxylipin Profiles in Humans. J Clin Endocrinol Metab 2020, 105(7).

41. Oka T, Fujimoto M, Nagasaka R, Ushio H, Hori M, Ozaki H: Cycloartenyl ferulate, a component of rice bran oil-derived gamma-oryzanol, attenuates mast cell degranulation. Phytomedicine 2010, 17(2):152-156.

42. Islam MS, Murata T, Fujisawa M, Nagasaka R, Ushio H, Bari AM, Hori M, Ozaki H: Anti-inflammatory effects of phytosteryl ferulates in colitis induced by dextran sulphate sodium in mice. Brit J Pharmaco/2008, 154(4):812-824.

43. Reitzer L: Biosynthesis of Glutamate, Aspartate, Asparagine, L-Alanine, and D-Alanine. EcoSal Plus 2004, 1(1).

44. Sakai R, Ooba Y, Watanabe A, Nakamura H, Kawamata Y, Shimada T, Takumi A, van Goudoever JB, Narita T: Glutamate metabolism in a human intestinal epithelial cell layer model. Amino Acids 2020, 52(11-12):1505-1519.

45. Nong F, Luo S, Liang $Y$, Zhao Z, Xing S, Wen B, Zhou L: Evaluation of the effect of Dahuang-Mudan decoction on TNBS-induced colitis using UPLC-QTOF/MS-based metabolomic analysis. Biomed Chromatogr 2021, 35(3):e5003.

46. Bader JE, Enos RT, Velázquez KT, Carson MS, Nagarkatti M, Nagarkatti PS, Chatzistamou I, Davis JM, Carson JA, Robinson CM et at: Macrophage depletion using clodronate liposomes decreases tumorigenesis and alters gut microbiota in the AOM/DSS mouse model of colon cancer. Am J Physiol Gastrointest Liver Physiol 2018, 314(1):G22-g31.

47. Tjalsma $\mathrm{H}$, Boleij $\mathrm{A}$, Marchesi JR, Dutilh BE: A bacterial driver-passenger model for colorectal cancer: beyond the usual suspects. Nat Rev Microbio/2012, 10(8):575-582.

48. Ley RE, Turnbaugh PJ, Klein S, Gordon Jl: Microbial ecology: human gut microbes 
associated with obesity. Nature 2006, 444(7122):1022-1023.

49. Zhong N, Ma Y, Meng X, Sowanou A, Wu L, Huang W, Gao Y, Pei J: Effect of Fluoride in Drinking Water on Fecal Microbial Community in Rats. Biol Trace Elem Res 2021. 
Figures

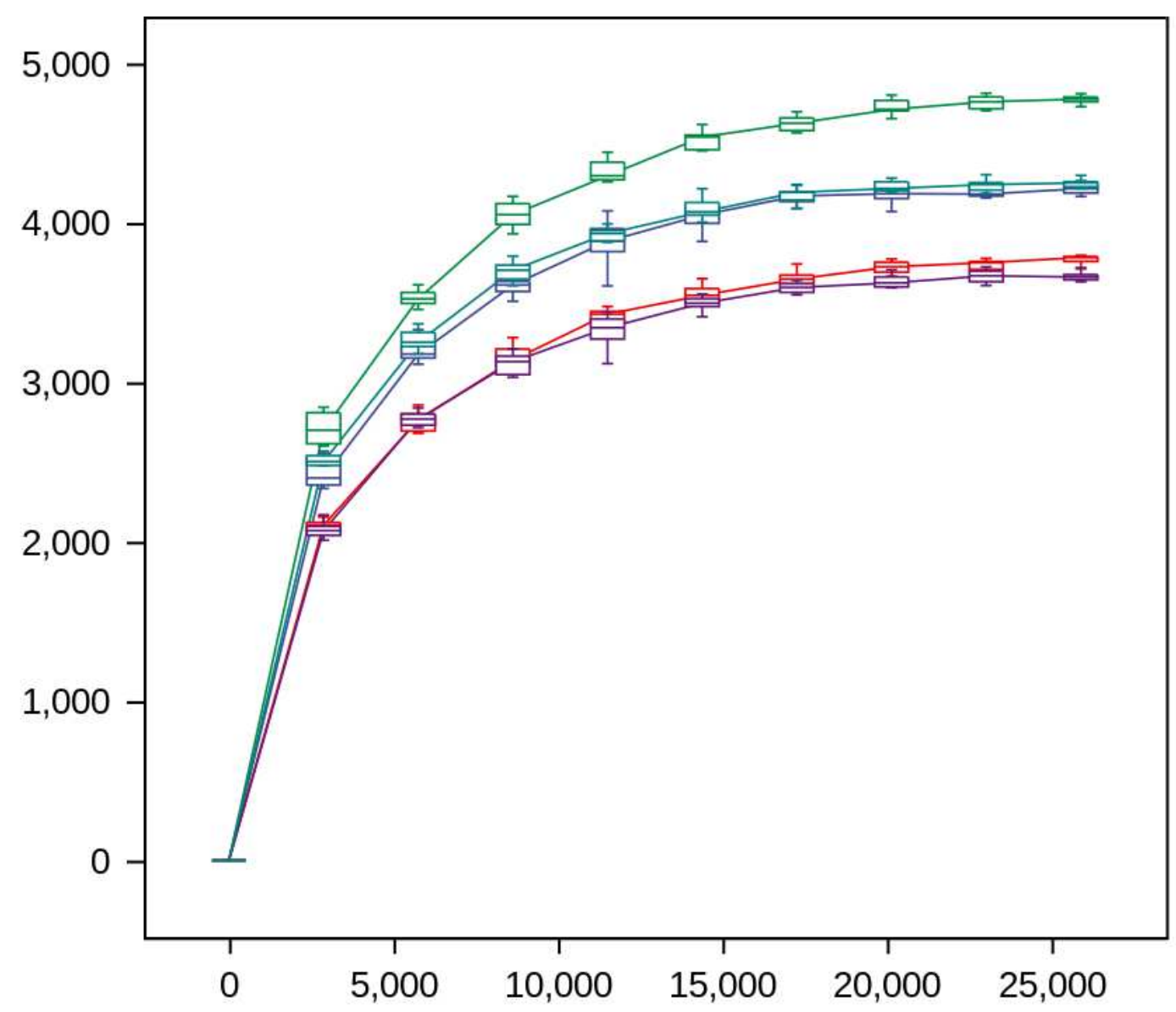

\section{Figure 1}

The rarefaction curves of each group based on Chao 1 


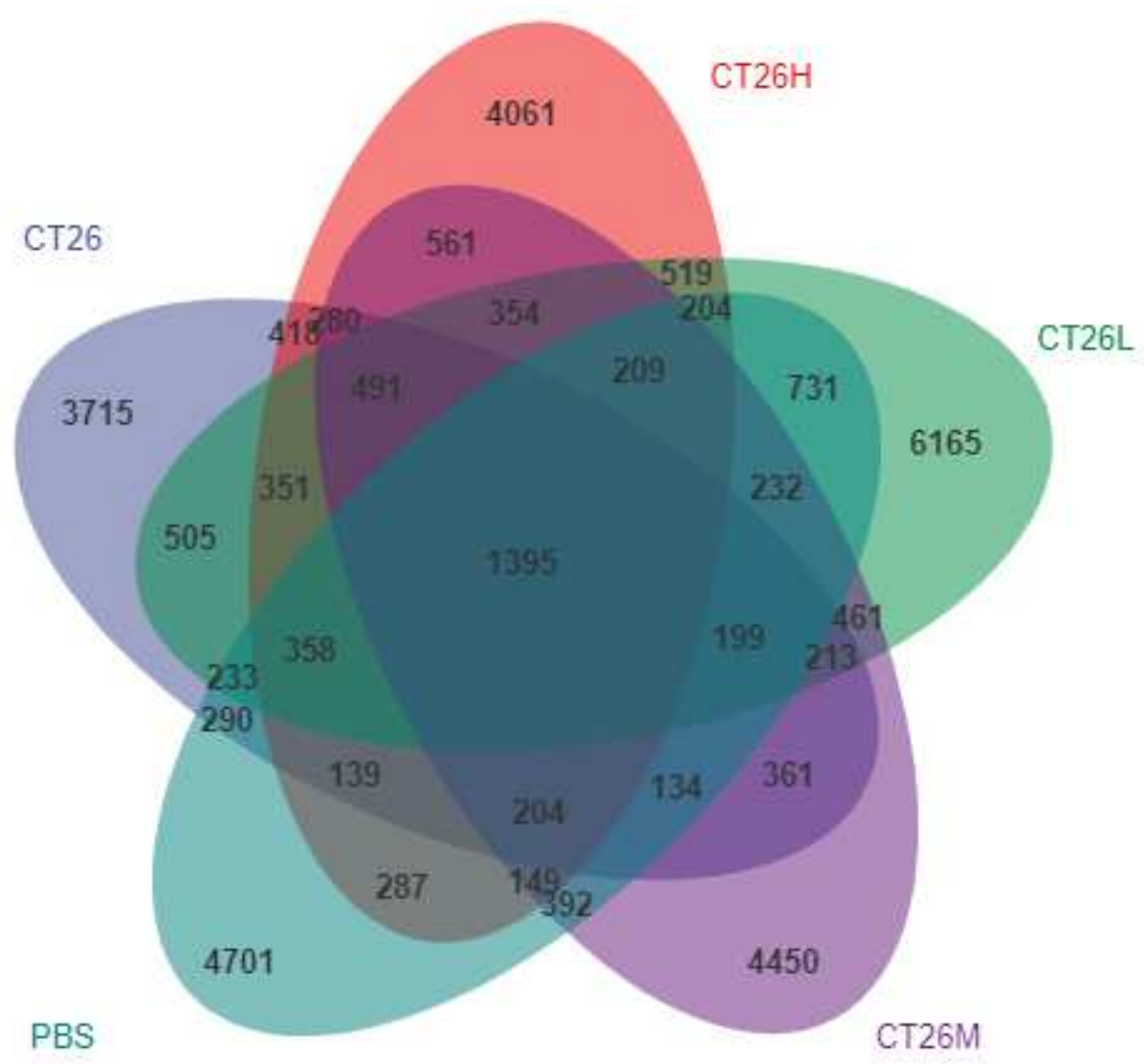

Figure 2

Venn diagram of OTUs in each group 


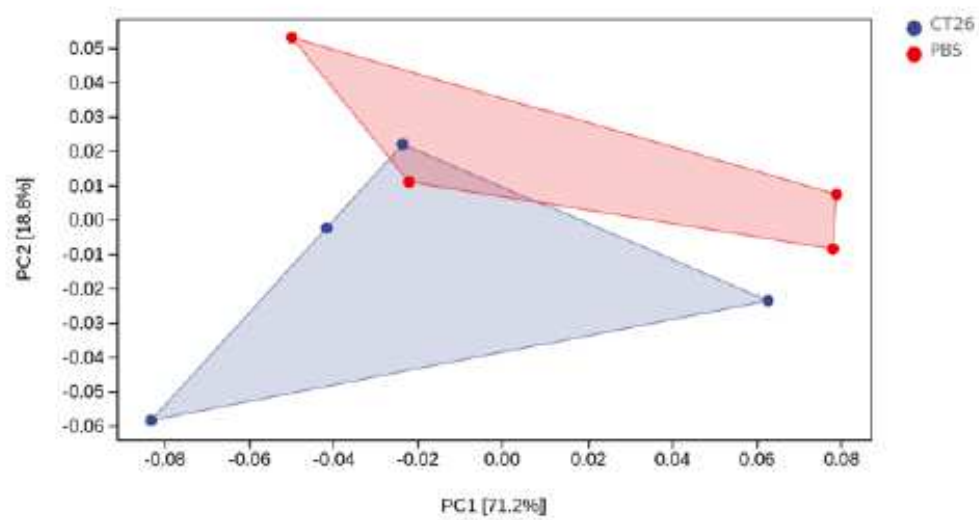

(a)

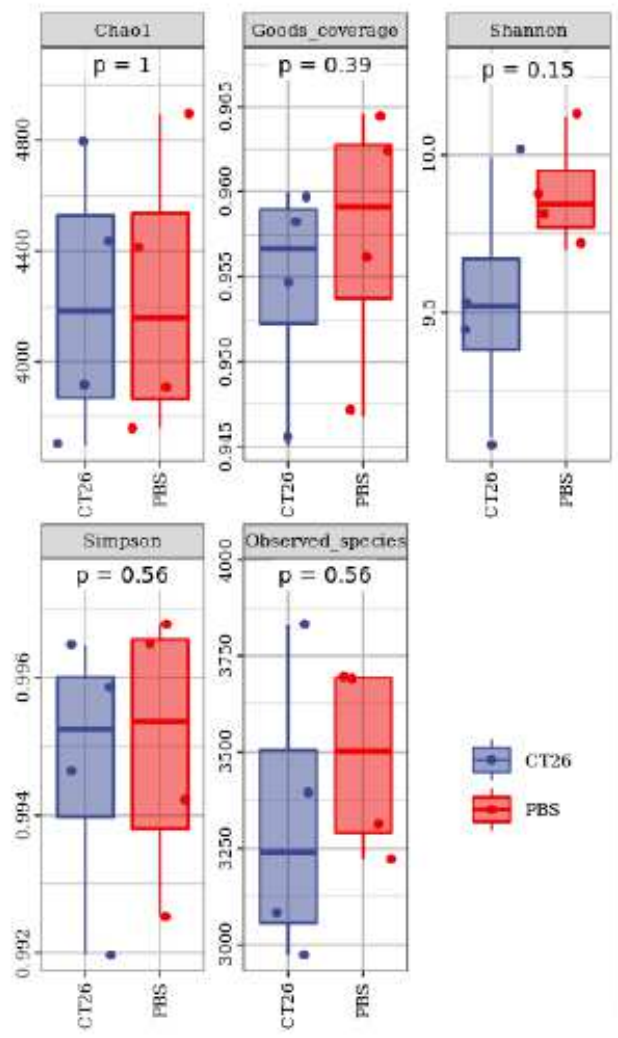

(c)

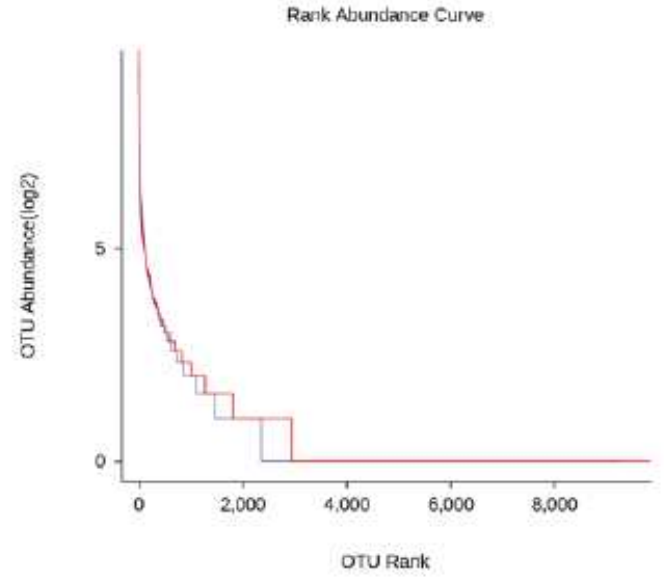

(b)

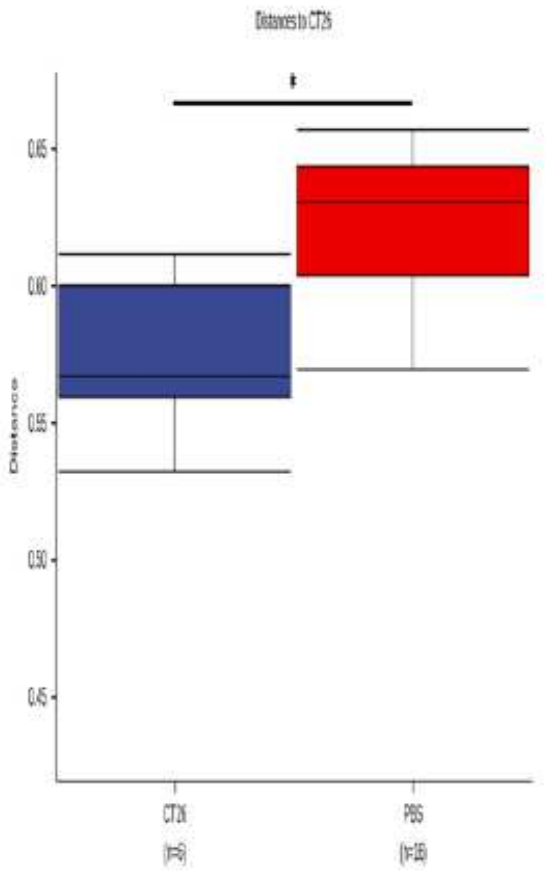

(d)

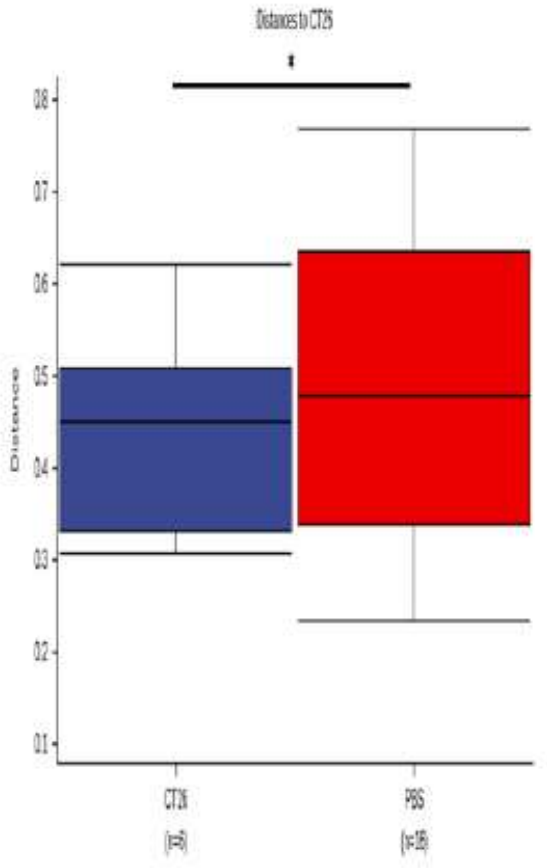

(e)

\section{Figure 3}

(a) The PCA diagram of CT26 group and PBS group. (b) The abundance grade curve of CT26 group and PBS group. (c) The differences in Chao1, Good's coverage, Shannon, Simpson and Observed species indices between CT26 group and PBS group. (d) The differences in unweighted UniFrac distance between CT26 group and PBS group. (e) The differences in weighted UniFrac distance between CT26 group and PBS group. 


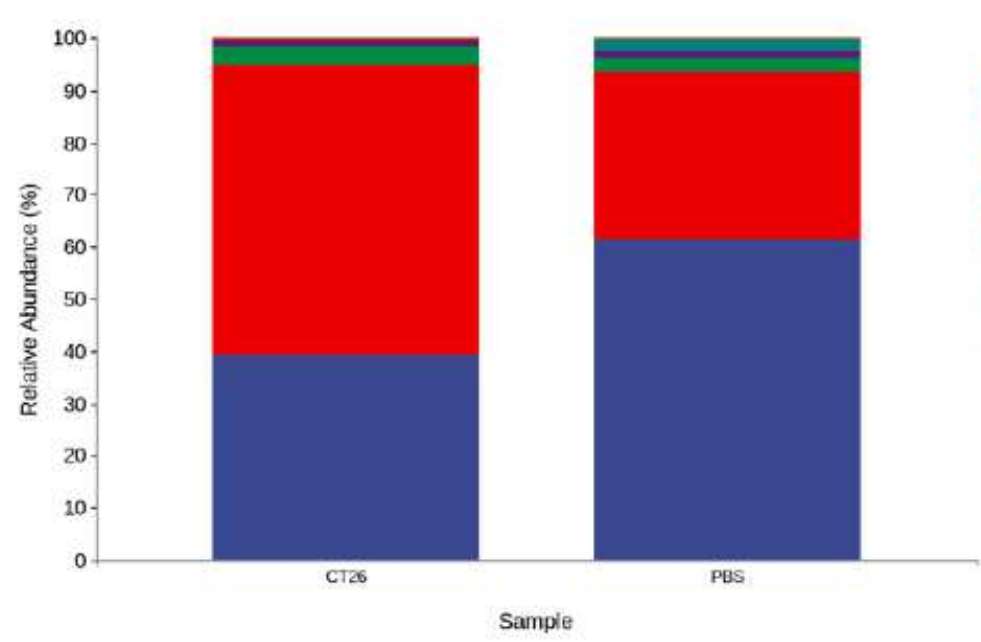

(a)

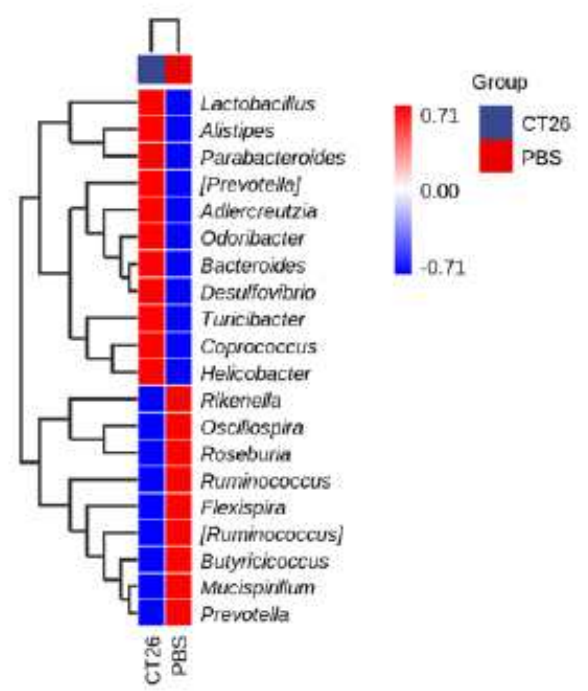

(b)

\section{Figure 4}

(a) Taxonomic summary of the gut microbiota at phylum level. (b) The heat map of species composition of the gut microbiota in CT26 group and PBS group at genus level.

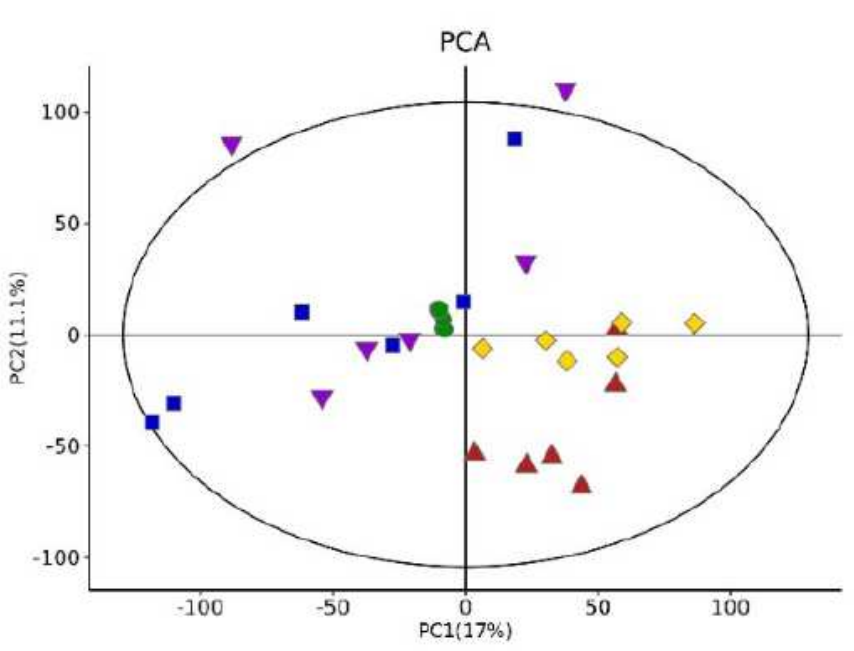

(a)
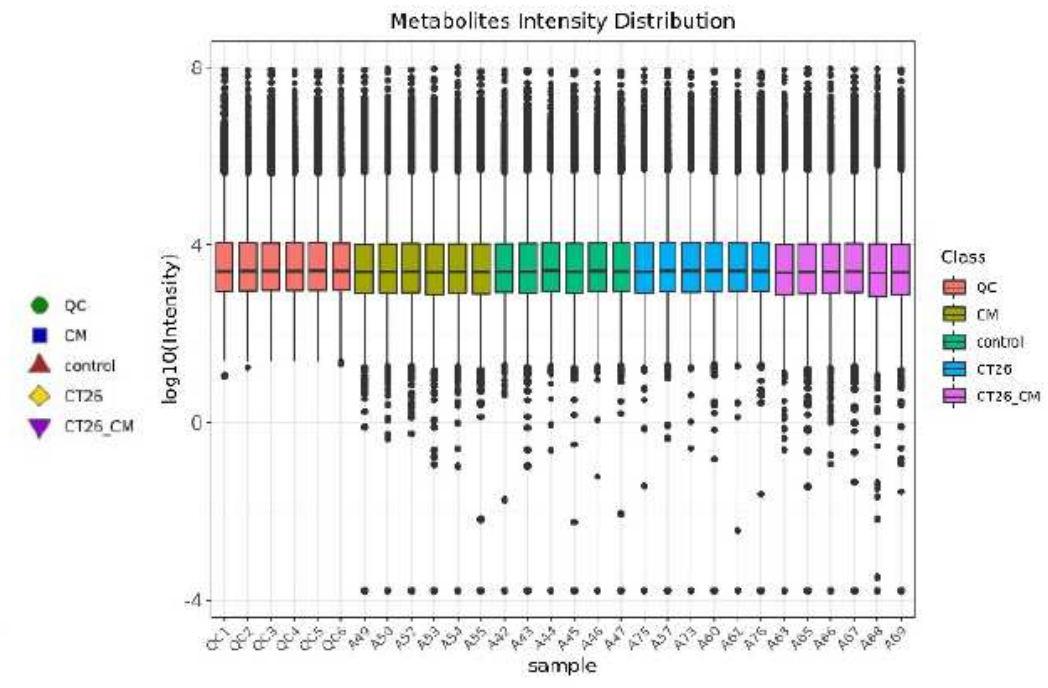

(b)

\section{Figure 5}

Quality control chart. (a) PCA score plot of all samples. (b) Metabolites Intensity Distribution box plot of all samples. 


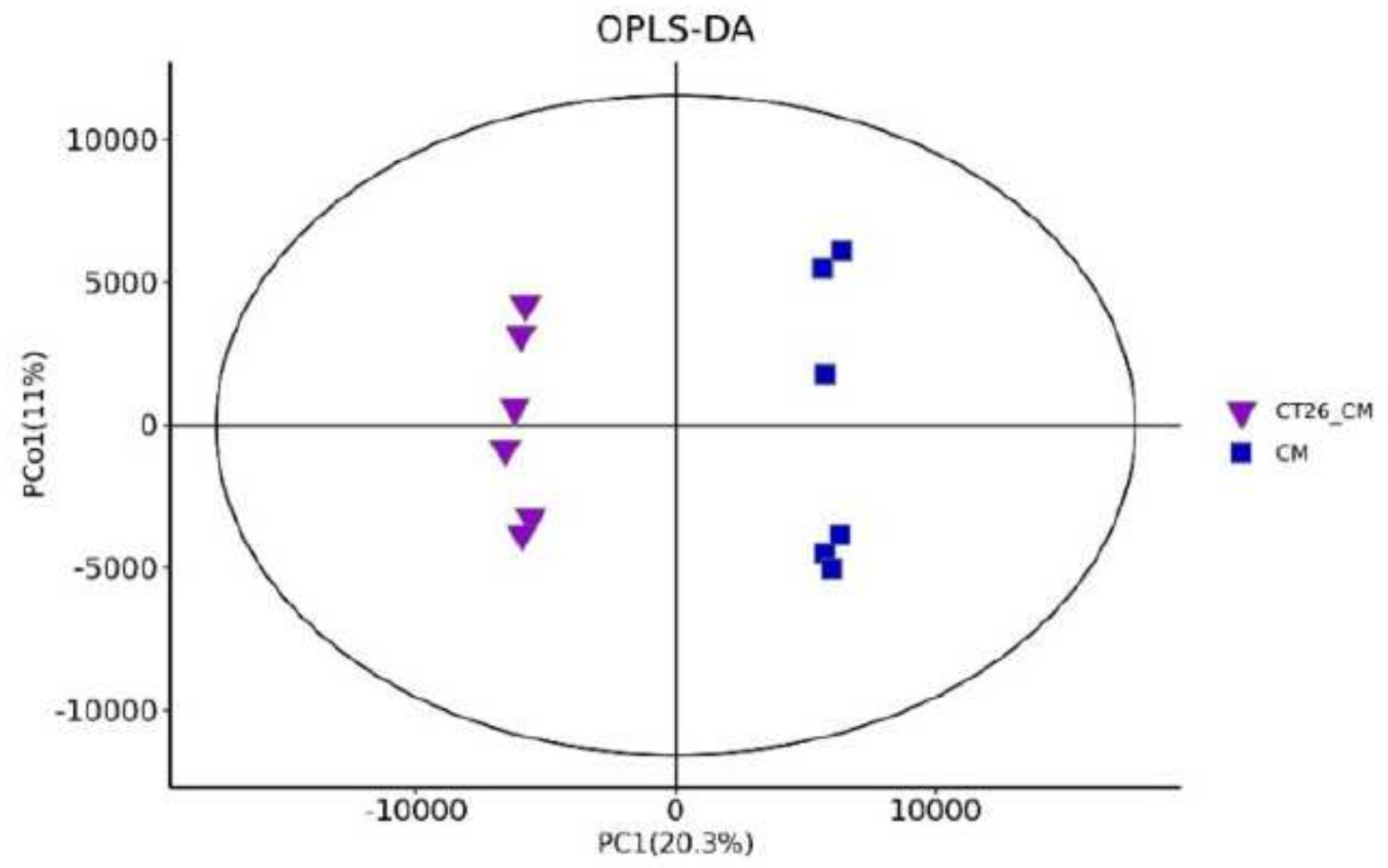

Figure 6

OPLS-DA score plot of tumor-bearing group treating by FFCT (CT26-CM group) and FFCT-treating group (CM group). $\mathrm{R} 2=(0.0,0.981), \mathrm{Q} 2=(0.0,-0.159)$.

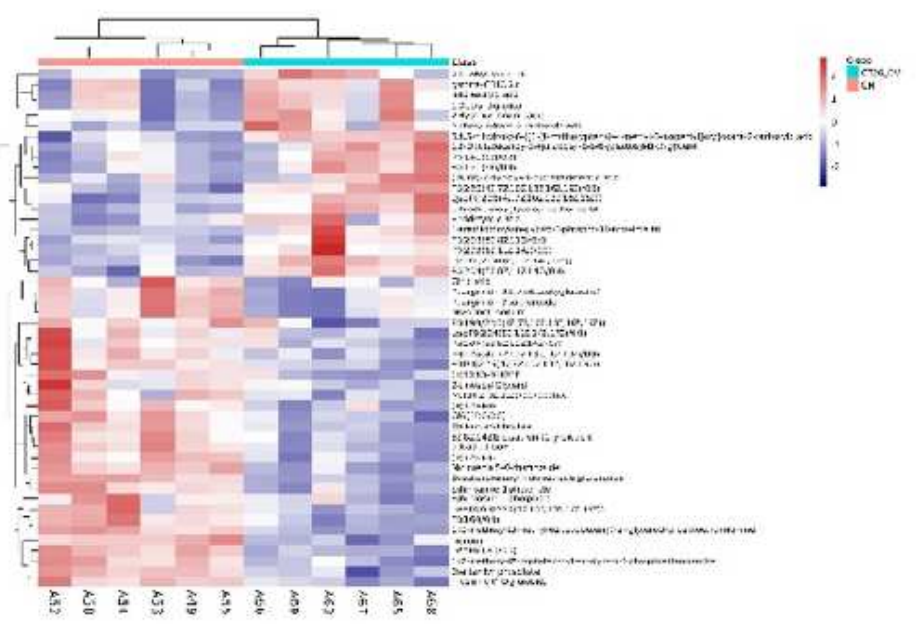

(a)

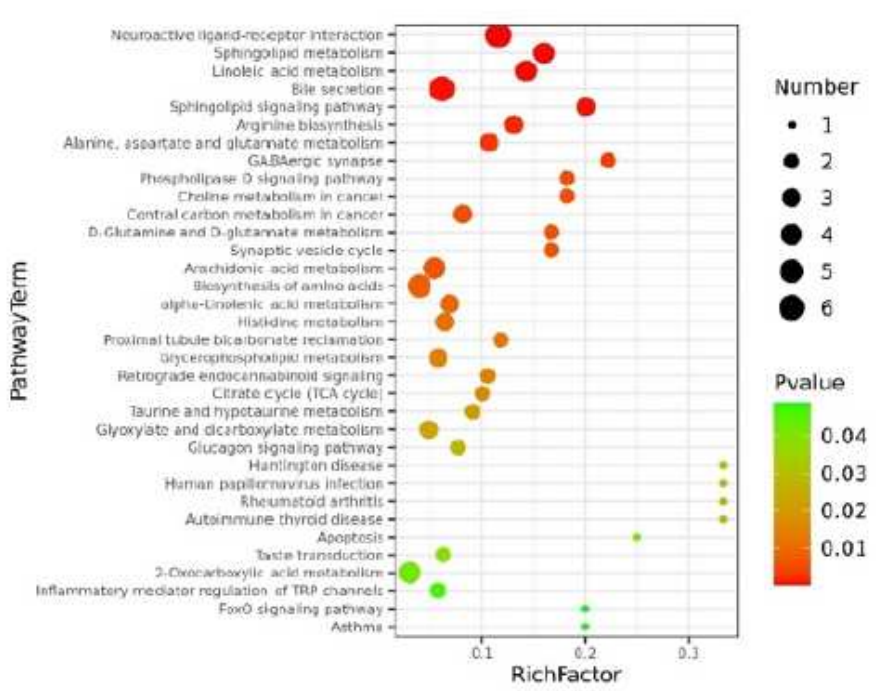

(b)

\section{Figure 7}

The hierarchical clustering heatmaps. (a) The hierarchical clustering heatmap of different metabolites in CT26-CM group and CM group. (b) KEGG pathways enriched by significantly different metabolites 
between CT26-CM group and CM group $(\mathrm{p}<0.05)$.

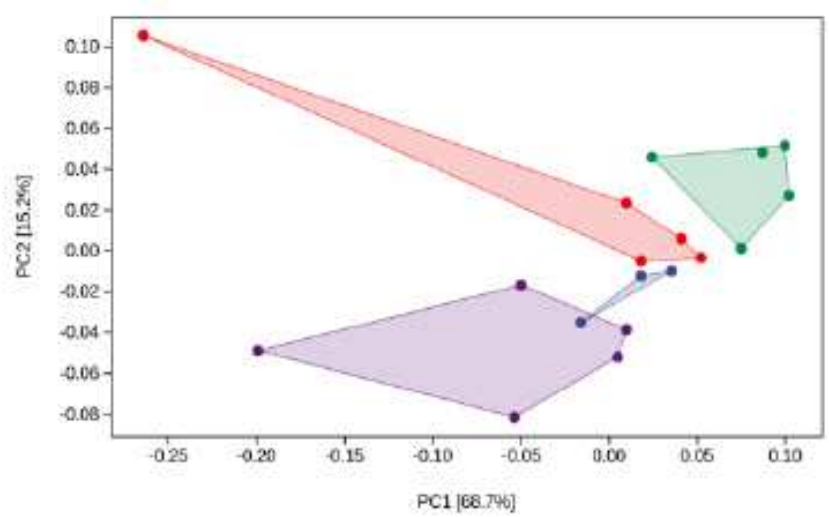

(a)

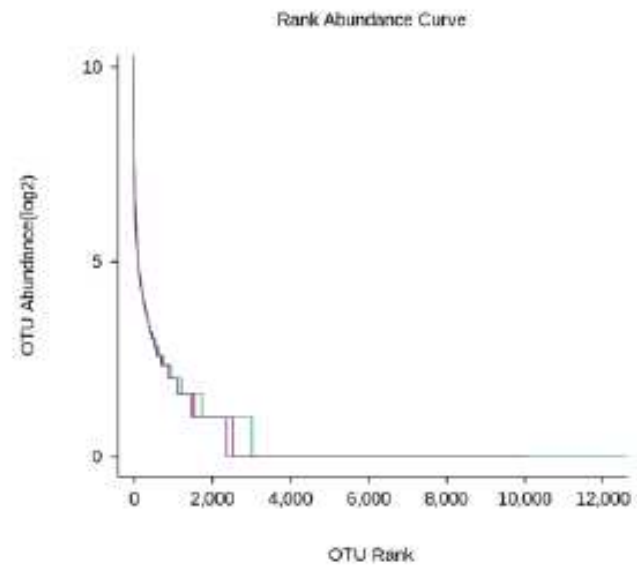

(b)

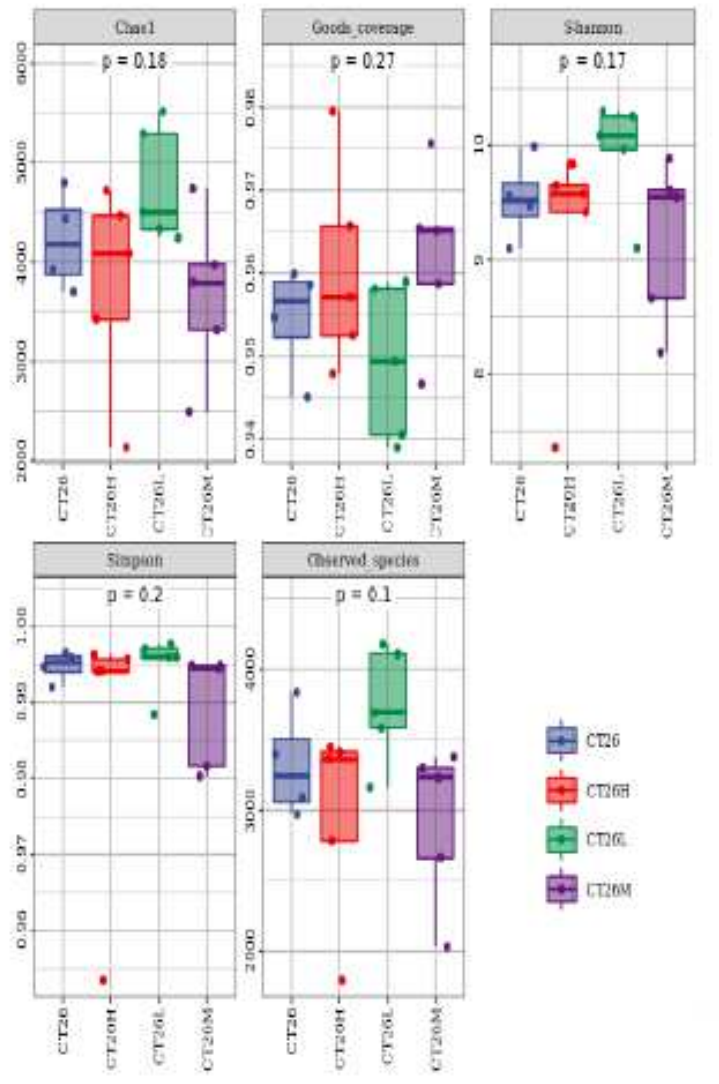

(c)

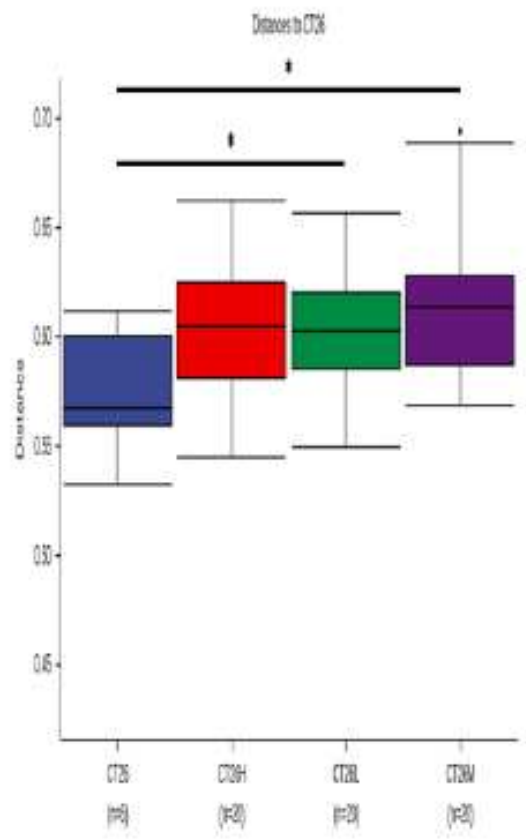

(d)

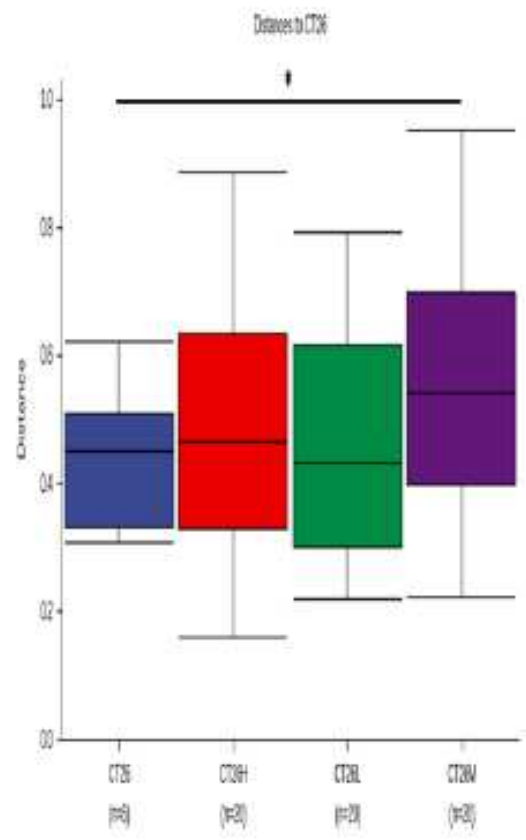

(e)

\section{Figure 8}

(a) The PCA diagram of CT26 group and FFCT-treating groups. (b) The abundance grade curve of CT26 group and FFCT-treating groups. (c) The differences in Chao1, Good's coverage, Shannon, Simpson and Observed species indices between CT26 group and FFCT-treating groups. (d) The differences in 
unweighted UniFrac distance between CT26 group and FFCT-treating groups. (e) The differences in weighted UniFrac distance between CT26 group and FFCT-treating groups.

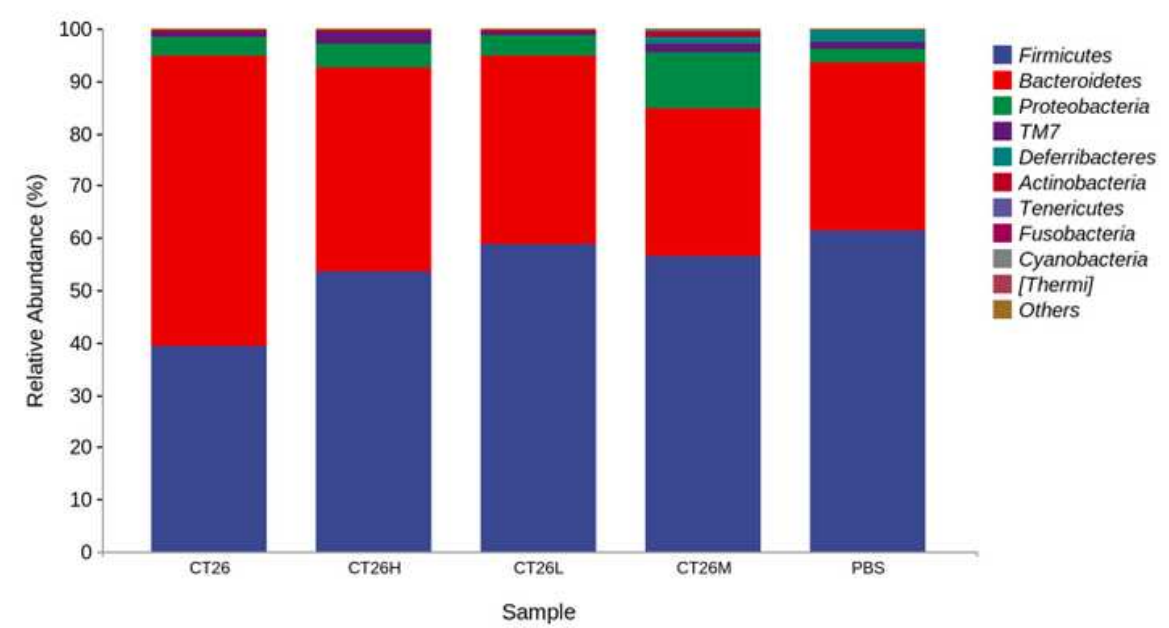

(a)

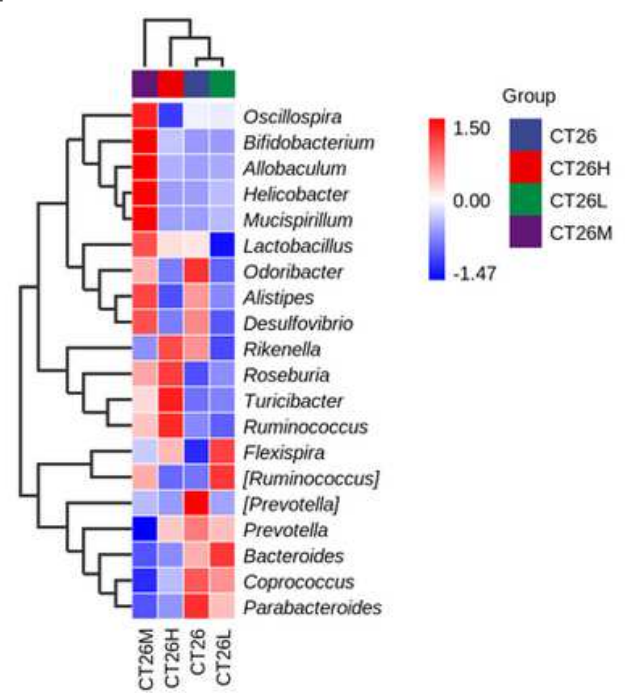

(b)

\section{Figure 9}

(a) Taxonomic summary of the gut microbiota at phylum level. (b) The heat map of species composition of the gut microbiota in CT26 group and FFCT-treating groups at genus level.

\section{Supplementary Files}

This is a list of supplementary files associated with this preprint. Click to download.

- T1unweightUnifracCT26PBS.xls.xlsx

- T2weightUnifracCT26PBS.xIs.xIsx

- T3unweightUnifracCT26CM.xls.xlsx

- T4weightUnifracCT26CM.xls.xIsx 